\title{
Title: Sequential establishment of stripe patterns in an expanding cell population
}

One-sentence summary: A synthetic circuit implementing density-controlled motility autonomously produces a tunable number of stripes.

\section{Authors and their affiliated institutions:}

Chenli Liu ${ }^{1 \ddagger}$, Xiongfei Fu ${ }^{2 \ddagger}$, Lizhong Liu ${ }^{1}$, Xiaojing Ren ${ }^{3}$, Carlos K.L. Chau ${ }^{1}$, Sihong $\mathrm{Li}^{2}$, Lu Xiang ${ }^{1}$, Hualing Zeng ${ }^{2}$, Guanhua Chen ${ }^{3}$, Lei-Han Tang ${ }^{4}$, Peter Lenz ${ }^{5}$, Xiaodong Cui ${ }^{2}$, Wei Huang ${ }^{1,2 *}$, Terence Hwa ${ }^{6}$, Jian-Dong Huang ${ }^{1}$ *

${ }^{1}$ Department of Biochemistry, ${ }^{2}$ Department of Physics, and ${ }^{3}$ Department of Chemistry, The University of Hong Kong, Pokfulam, Hong Kong, China

${ }^{4}$ Department of Physics, Hong Kong Baptist University, Kowloon Tong, Kowloon, Hong Kong, China

${ }^{5}$ Department of Physics and Center for Synthetic Microbiology, University of Marburg, 35032 Marburg, Germany

${ }^{6}$ Center for Theoretical Biological Physics, University of California at San Diego, La Jolla, CA, USA

${ }^{\ddagger}$ These authors contributed equally to this work.

*Correspondence should be addressed to JDH (jdhuang@hku.hk), TH (hwa@ucsd.edu), or WH (huangwei@hku.hk) 
Periodic stripe patterns are ubiquitous in living organisms, yet the underlying developmental processes are complex and difficult to disentangle. We describe a synthetic genetic circuit that couples cell density and motility. This system enabled programmed Escherichia coli cells to form periodic stripes of high- and low- cell densities sequentially and autonomously. Theoretical and experimental analyses reveal that the spatial structure arise from a recurrent aggregation process at the front of the continuously expanding cell population. The number of stripes formed could be tuned by modulating the basal expression of a single gene. The results establish motility control as a simple route to establishing recurrent structures without requiring an extrinsic pacemaker. 
Living organisms display an amazing array of regular spatial patterns (1-4). Traditionally, elucidation of their developmental mechanisms is pursued through forward or reverse genetics (3, 5). However, essential components required for pattern formation and control are often buried in the overwhelmingly complex physiological context. Synthetic biology provides an engineering approach to examine strategies of pattern formation (6-8). Recently, efforts have been made to emulate patterning systems using pre-deposited positional cues $(9,10)$. These studies do not address spatial or temporal self-organization, which is key to morphogenesis. Indeed, coordinated cell movement in response to self-generated cues is important in embryonic development (11-13). Here, we investigate striking patterns that emerge in growing bacterial populations when cell motility is controlled by cell density, construed as one of the simplest examples of morphogenic cues (14).

We constructed a genetic circuit to suppress the motility of Escherichia coli cells at high cell densities (Fig. 1A). The circuit is composed of two modules: a density-sensing module and a motility-control module (Fig. 1B). The quorum-sensing system in Vibrio fischeri was adopted as the density-sensing module to signal the local cell density. This system synthesizes and excretes a small molecule acyl-homoserine lactone (AHL), which, at high extracellular levels (reflecting high cell density), accumulates intracellularly and activates a constitutively expressed regulator, LuxR (15). The motility-control module was devised to modify bacterial motility by regulating the transcription of cheZ. CheZ deletion causes cells to tumble incessantly, resulting in a non-motile phenotype in semi-solid agar (16). Reintroducing cheZ restores cell motility (17). In our circuit, coupling of the two modules was achieved via the lambda repressor (CI): the LuxR/AHL complex drove the expression of $\mathrm{CI}$ that in turn repressed cheZ transcription (Fig. 1B). Based on this design, we created an engineered strain CL3 (18).

Gene expression of the strain CL3 at various cell densities were quantitatively characterized using qRT-PCR. As designed, cI expression level (red) elevated over 40-fold as cell density 
increased whilst cheZ expression (blue) decreased sharply to 5\% of the high level (Fig. 1C). Cell motility was measured in semi-solid agar using a modified Continuous-Fluorescence-

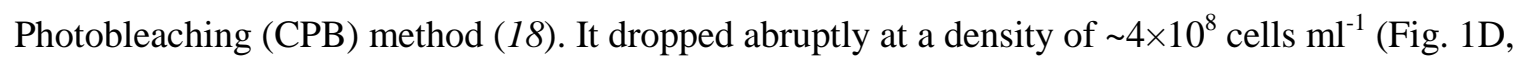
purple symbols).

When a suspension of exponentially growing CL3 cells was inoculated at the centre of a 8.5-cm Petri dish containing $10-\mathrm{ml}$ LB medium and $0.25 \%$ agar, a pattern of alternating white (high cell density) and dark (low cell density) stripes developed overnight (Fig. 1E, Movie S1) (18). The stripes formed sequentially, at a spacing of approximately $0.5 \mathrm{~cm}$ once every $\sim 2$ hours. These stripes were stable for days until the agar completely dried up. As a control, strain CL4 (carrying the density-sensing module but with the native cheZ regulation) behaved like wild type (16): from the position of the inoculum, two traveling waves successively moved radially outwards, followed by a uniform expansion of cell density without stripes (Fig. 1F and Movie S2). We further verified that each element in the designed genetic circuit is necessary for the stripe pattern formation (Fig. S1). We also surveyed the effect of nutrient content, agar thickness, humidity, starting cell number, volume, and growth phase, and found the pattern to be robust to most conditions tested (Fig. S2). Importantly, the stripe pattern persists even in $c h e B^{-}{ }^{c} h R^{-}$double mutants incapable of chemotaxis [Fig. S2J, (19)] but still able to swim and tumble randomly, indicating that the stripes are not a result of chemotactic signaling (20). Also, no stripe pattern resulted if CL3 cells were spotted on agar pre-mixed with a low level of CL3 cells in otherwise identical conditions (Fig. S2K), indicating that the pattern is not a result of spontaneous amplification of density fluctuations, as would follow from the paradigm of Turing instability $(21,22)$.

We monitored the spatiotemporal cell density profiles from optical absorbance of the plates [Fig. S3 and (18)]. Fig. 1G displays the time course of light intensities along a diagonal that goes through the inoculum, with the color codes indicating the intensity as in Fig. 1E. It shows that once 
formed, the pattern of low and high cell densities was frozen in space. Furthermore, new stripes of high cell density emerged at a regular time and distance intervals. The same phenomenon was observed in a rectangular geometry when bacterial growth was initiated along a thin line in the middle of the plate (18). Stripes developed and propagated towards the opposite edges for CL3 (Fig. S4A and Movie S3) but not for the wild type or CL4 cells. Fourier analysis revealed high spatial periodicity, with a wavelength of $0.5 \mathrm{~cm}$ (Fig. S4B).

To gain a quantitative understanding of the patterning process, we developed a mathematical model based on the characterized properties of the engineered circuit [Fig. 2 and (18)]. The stochastic swim-and-tumble motion of bacterial cells is described at the population level by a diffusion-like equation (Eq. [1]) for the cell density $\rho(x, t)$. The synthesis, diffusion, and turnover of AHL are described by Eq. [2], while the consumption and diffusion of the nutrient $n(x, t)$ are described by Eq. [3]. The AHL-dependent motility enters through a phenomenological diffusion coefficient $\mu(h)$ that drops abruptly where the local AHL concentration $h(x, t)$ exceeds a threshold level $K_{h}$ (Fig. 2A). Numerical simulations of Eqs. [1]-[3] (23) using realistic parameter values [Table S3 and (18)] generated patterns similar to the experimental observations in both one- and two- dimensional geometries (Fig. S5 and S20). The model also captures the more elaborate patterns formed by multiple seeding (Fig. S6).

The mathematical model reveals an intriguing sequence of events at the propagating front (Fig. 2B and Movie S4). Early on ( $\mathrm{T}=150 \mathrm{~min}$ ), cell density (blue line) grows and propagates laterally just as the Fisher wave front (2) since the AHL concentrations (red line) is uniformly below the threshold level $K_{h}$ (grey dashed line) due to the low initial cell density. A while later ( $\mathrm{T}=300 \mathrm{~min}$ ), the AHL level exceeds the threshold in a region behind the front due to local cell proliferation: there, a "bud” (marked by B) shoots up in the density profile. Meanwhile, the front (marked by F), which is unaffected by what happens behind due to its low density, continues to 
propagate laterally at the same speed. Later $(\mathrm{T}=450 \mathrm{~min})$, the bud in the density profile grows into a “mound” (marked by M), separated from the frontal region by a "cleft” (marked by C). The continued cell proliferation in the region just behind the front drives up the AHL level and subsequently produces a new bud (B' at $T=600 \mathrm{~min}$ ), while the lateral movement of the mound and cleft eventually stops ( $\mathrm{M}$ and $\mathrm{C}$ at $\mathrm{T}=600 \mathrm{~min}$ ). The new bud starts a new stripe of low motility cells which again expands into a mound ( $\mathrm{M}^{\prime}$ at $\mathrm{T}=750 \mathrm{~min}$ ) delimited by a cleft ( $\mathrm{C}^{\prime}$ at $\mathrm{T}=750 \mathrm{~min}$ ) from the propagating front, and the process repeats itself. Well behind the front, cell proliferation in both the mound and cleft eventually stops as the nutrient is depleted (height of green shade).

The development of a cleft that separates the propagating front from the dense zone of cells behind, and the budding within the frontal region result from an aggregation phenomenon driven by density-dependent motility discussed in recent theoretical studies $(21,24)$ and illustrated in Fig. 3A. Cells can diffuse freely in semi-solid agar when the cell density is low (top panel). As cells proliferate and the local AHL level exceeds the threshold, cell motility slows down as programmed (middle panel, with the non-motile cells in teal). These cells cannot move away but neighboring cells may continue to move into this high-density region and become non-motile (bottom panel), leading to a net cell-flow towards the high-density region.

Previously, it has been reported that wild-type E. coli cells may aggregate when grown in specific poor-nutrient media due to the secretion of potent chemo-attractants (25). To show that cell aggregation in our system is controlled by the synthetic circuit, we created two strains, the senders (CL6) that synthesize AHL but are non-motile, and the receivers (CL8), capable of receiving AHL and regulating motility but incapable of AHL synthesis. The receiver cells were uniformly mixed with semi-solid agar. Subsequently, a drop of sender cells was spotted at the center of the hardened cell-agar mixture. The non-motile sender cells could not move away from where they were spotted, but were expected to synthesize and excrete AHL as they proliferated at the center of the plate. 
After 12-h incubation, a high-density stripe of receiver cells aggregated around the spotted sender cells (Fig. 3B upper panels, Movie S5). No aggregation was observed in a control with receiver cells incapable of reducing motility in response to high AHL concentration (CL10) (Fig. 3B lower panels), nor in other controls (Fig. S7). Thus, an effective aggregation phenomenon was mediated by density-dependent motility.

Detailed analysis of the mathematical model indicates that the aggregation effect alone is not sufficient to generate stripes. Budding in the frontal region requires the AHL level to exhibit a local maximum, which can happen only when the diffusion length of AHL molecules is sufficiently short (so that the AHL profile closely follows the cell density profile), and the cleft in the cell density profile just behind the front is sufficiently deep. The latter is determined in turn by the parameters, such as the maximum cell motility $(18,26)$. A key output of the model is summarized by the phase diagram (Fig. 4A), which predicts that in addition to the periodic stripe phase, the engineered strain may exhibit a qualitatively different behavior with no stripes, by passing through a transition region exhibiting a limited number of stripes.

A direct test of the occurrence of the phase transition predicted by the phase diagram (Fig. 4A) is to vary the maximum cell motility ( $D_{\rho}$ in Fig. $\left.2 \mathrm{~A}\right)$, thereby changing the number of stripes. Within our experimental design in which motility is set by cheZ expression, tuning of $D_{\rho}$ can be implemented by adding a second $c I$ gene, whose expression is titratable in an AHL-independent manner (Fig. 4B). This is implemented in strain CL5 (18). Fig. 4C shows that the basal $c I$ expression level of CL5 cells could indeed be smoothly tuned by adjusting the dosage of an inducer, anhydrotetracycline (aTc), at a fixed cell density (red symbols). Corresponding suppression of cheZ expression at low cell density was also observed (blue symbols). For a fixed aTc level, the density dependence of cheZ expression remained (Fig. S8). When strain CL5 was spotted at the center of semi-solid agar, consistent with the model predictions, the number of stripes decreased gradually as 
the aTc concentration increased from 0.1 to $3.0 \mathrm{ng} \mathrm{ml}^{-1}$ (Fig. 4D). As a control, the pattern of strain CL3 did not change even when the aTc concentration was increased to $100 \mathrm{ng} \mathrm{ml}^{-1}$ (Fig. S2G).

Natural occurrences of well-coordinated spatial and/or temporal patterns are abundant in developmental systems, and are believed to involve elaborate control mechanisms $(3,5)$. Similarly, stripe formation in various bacterial systems (27-29) has been attributed to complex effects involving chemotaxis, swarming, and differentiation (4). Using synthetic circuits, we demonstrate that precise and robust spatiotemporal patterns can be generated autonomously from a very simple interaction - motility control by density. A recurrent mechanism enables structures to form periodically and sequentially. Important features of the pattern such as the number of stripes can be manipulated by tuning components of the circuit, such as the basal expression level of a single gene.

The strategy of sequential stripe formation is likely not limited to cellular motility control. In the kinetic equations [1]-[3] that generated the stripe patterns, the cell density $\rho(x, t)$ is merely an example of space- and time- dependent variable. Another example could be the concentration of a diffusible molecule, which stimulates its own synthesis to mimic the effect of “cell growth”. Thus Eqs. [1]-[3] may be taken as a generalized reaction-diffusion system with the key feature that the mobility or transport of one of the regulators between adjacent cells is regulated by another mobile regulator. As regulated mobility of signaling molecules is a common strategy in metazoan development $(30,31)$, the lessons from this work may stimulate new insights and inspire new directions in the studies of developmental systems. For example, the sequential establishment of a periodic somite structure during vertebrate embryonic development is commonly assumed to be controlled by an enabling clock coupled to a sweeping morphogen gradient (32). Our results suggest that a spatially periodic structure can be formed autonomously without the need for a clock. 


\section{References and Notes}

1. L. I. Held, Models for embryonic periodicity. (Karger, Basel, 1992), pp. viii, 119 p.

2. J. D. Murray, Mathematical biology. I., An introduction. (Springer, New York, ed. 3rd, 2002), pp. xxiii, 551 p.

3. $\quad$ C. M. Chuong, M. K. Richardson, Int J Dev Biol 53, 653 (2009).

4. $\quad$ E. Ben-Jacob, I. Cohen, H. Levine, Adv Phys 49, 395 (2000).

5. $\quad$ E. H. Davidson, D. H. Erwin, Science 311, 796 (2006).

6. A. S. Khalil, J. J. Collins, Nat Rev Genet 11, 367 (2010).

7. $\quad$ M. Elowitz, W. A. Lim, Nature 468, 889 (2010).

8. $\quad$ S. Mukherji, A. van Oudenaarden, Nat Rev Genet 10, 859 (2009).

9. $\quad$ S. Basu, Y. Gerchman, C. H. Collins, F. H. Arnold, R. Weiss, Nature 434, 1130 (2005).

10. M. Isalan, C. Lemerle, L. Serrano, PLoS Biol 3, e64 (2005).

11. P. Rorth, Trends Cell Biol 17, 575 (2007).

12. D. J. Montell, Curr Opin Genet Dev 16, 374 (2006).

13. A. Aman, T. Piotrowski, Dev Biol 341, 20 (2010).

14. L. E. Weiss et al., Biotechnol Bioeng 100, 1251 (2008).

15. C. M. Waters, B. L. Bassler, Annu Rev Cell Dev Biol 21, 319 (2005).

16. A. J. Wolfe, H. C. Berg, Proc Natl Acad Sci U S A 86, 6973 (1989).

17. M. G. Sanna, M. I. Simon, J Bacteriol 178, 6275 (1996).

18. Materials and methods are available as supporting material on Science Online.

19. J. S. Parkinson, S. E. Houts, J Bacteriol 151, 106 (1982).

20. H. C. Berg, Annu Rev Biophys Bioeng 4, 119 (1975).

21. M. E. Cates, D. Marenduzzo, I. Pagonabarraga, J. Tailleur, Proc Natl Acad Sci U S A 107, 11715 (2010).

22. $\quad$ A. M. Turing, Philos Trans R Soc Lond B Biol Sci 237, 37 (1952).

23. We believe Eqs. [1]-[3] comprise the minimalistic, experimentally faithful model needed to generate the observed patterns. A simplified model in which the explicit description of nutrient dynamics was replaced by a growth saturation term (Eq. [S11]) was able to initiate but not maintain the patterns, since cell density eventually took on the saturation value everywhere in space (Fig. S17A). A related density-only model (21) generated stripe patterns by balancing cell aggregation with cell death [see also (18)]; it is not applicable to our experiments since cells stop growing but do not die on the relevant time scales. The density-only model also predicted patterns to form from small initial density fluctuation, in contrast to our observation (Fig. S18) and model output (18).

24. J. Tailleur, M. E. Cates, Phys Rev Lett 100, 218103 (2008).

25. E. O. Budrene, H. C. Berg, Nature 349, 630 (1991).

26. Eqs. [1]-[3] can produce a variety of stripe patterns depending on the values of two key parameters, maximum motility $D_{\rho}$ and AHL half life $\operatorname{Ln}(2) / \beta$, as summarized in the phase diagram (Fig. 4A), see (18) for details. To the right of the solid red line, the system exhibits the periodic stripe phase with an infinite number of stripes. Moving to the left of the solid line, there is a regime where the system can still generate a limited number of stripes for some initial conditions. Far away from the solid line no stripes can form. The separation between the latter two regimes is not clear-cut and is indicated by the dashed green line. Additional effects such as chemotaxis (Eq. [S17]) have been investigated. They modify the detailed appearances of the patterns as well as the location of the phase boundaries (Fig. S21). Importantly, the general occurrence of the periodic stripes in this class of models does not require such effects.

27. H. Fujikawa, Physica A 189, 15 (1992). 
28. T. Matsuyama et al., J Bacteriol 182, 385 (2000).

29. D. E. Woodward et al., Biophys J 68, 2181 (1995).

30. L. Niswander, Nat Rev Genet 4, 133 (2003).

31. M. Affolter, K. Basler, Nat Rev Genet 8, 663 (2007).

32. O. Pourquie, Science 301, 328 (2003).

\section{Acknowledgments:}

We are grateful for the members of the University of Hong Kong Team for The International Genetic Engineering Machine Competition (iGEM) 2008 for their contribution to the project. We thank Howard Berg, Albert Courey, Julien Tailleur, and Chris Voigt for valuable comments. This project was supported by an HKU University Development Fund, an HKU CRCG Small Project grant and an RGC CRF (HKU1/CRF/10) to JDH, and a Faculty Development Fund to WH. TH acknowledges an HKU Distinguished Visiting Professorship. PL acknowledges support through the LOEWE program. LHT acknowledges support by the RGC of the HKSAR under grant 201606.

Supporting Online Material

www.sciencemag.org

Methods

Model

References (33-63)

Figs. S1 to S21

Tables S1 to S3

Movie S1 to S5 


\section{Figure Legends}

Fig. 1. Spatiotemporal patterns formed by engineered E. coli strains. (A) Illustration of the desired cell behavior. (B) Design of the genetic circuit; see text. (C) Relative cheZ and $c I$ mRNA level in strain CL3 as a function of cell density in bulk culture by qRT-PCR. Data are normalized by the mean value of cells at $0.3 \times 10^{8}$ cells $\mathrm{ml}^{-1}$. (D) The relative diffusion coefficient as a function of cell density. CL3GFP, strain CL3 carrying superfolder GFP. CL4 (Cn), strain CL4 as a control (carrying density-sensing module but with native cheZ regulation) (18). Relative diffusion coefficient values are normalized by the mean value of CL3GFP at $0.4 \times 10^{8}$ cells $\mathrm{ml}^{-1}$. Results are representative data from three independent experiments. Error bars represent s.d. of three replicates. Time-lapsed photographs of typical patterns obtained for the engineered strain CL3 (E) and the control strain CL4 (F); see also Movie S1 and S2. (G) Spatiotemporal diagram of (E). All experiments were carried out at $37^{\circ} \mathrm{C}$. Scale bars, $1 \mathrm{~cm}$.

Fig. 2. Kinetic model of autonomous periodic stripe pattern formation. (A) The model comprises three key ingredients, cell-density $(\rho)$, AHL concentrations $(h)$, and nutrient levels $(n)$, see text and (18). Crucial feature of this model is the AHL-dependent cell motility $\mu(h)$, in light of the data in Fig. 1D, is modeled by a steep Hill function (red line) with an abrupt transition between the values $D_{\rho}$ and $D_{\rho, 0}$ at $\underline{h \approx K_{h}}$ (dashed vertical line). (B) Time-lapse plots of the simulated relative cell-density profiles $\rho(x, t)$ (blue), AHL concentrations $h(x, t)$ (red), and nutrient levels $n(x, t)$ (green shade); see also Movie S4. The AHL threshold of motility regulation $K_{h}$ is shown as the horizontal dashed line; the vertical axis is rescaled to cell density $\left(10^{8}\right.$ cells $\left.\mathrm{ml}^{-1}\right)(18)$. The periodic pattern is seen as a recurrent process involving the formation of a bud (B), and the bud subsequent grows into 
a stationary mound (M) and a cleft (C) structure forms in a region between the bud and the expanding front.

Fig. 3. Cell aggregation driven by density-dependent motility. (A) Illustration of aggregation via density-dependent motility; see text. (B) Experimental evidence of effective aggregation. Receiver cells were uniformly mixed with $0.25 \%$ agar. 5 - $\mu$ l of sender cells (CL6, producing AHL but nonmotile, GFP-expressing) were spotted at the center of receiver-cell-agar mixture, followed by 12-h incubation at $37^{\circ} \mathrm{C}$. See (18) for details. Upper, strain CL8 (carrying the entire genetic circuit but lacking the AHL-producing gene) as receiver (Movie S5). Lower, strain CL10 (CL8 lacking cI) as receiver. Right panels, fluorescence photographs indicate the positions of the sender cells.

Fig. 4. Tunable stripe patterns. (A) Phase diagram; see (18) and (26). (B) Genetic circuit design for tuning stripe number. An aTc-inducible module was added to vary the expression of cheZ. (C) Relative cheZ and $c I$ mRNA level of CL5 in bulk culture containing various concentrations of aTc (0.1-3 ng $\mathrm{ml}^{-1}$ ). Cells were cultured to $\mathrm{OD}_{600} \sim 0.05$ and harvested for measurement. Data were

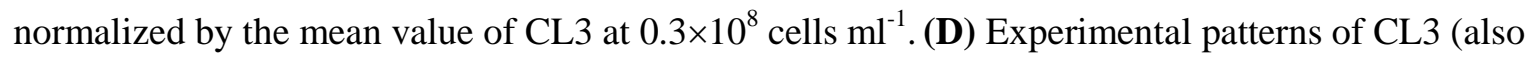
see Fig. S2G) or CL5 inoculated at $0.25 \%$ agar containing various concentrations of aTc. Agar plates were incubated at $37^{\circ} \mathrm{C}$ for $40 \mathrm{~h}$. 
A

\section{Stop}

Move

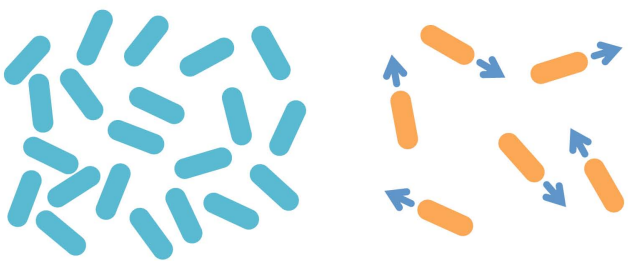

High density Low density
B ${ }^{\circ} \mathrm{HH}$

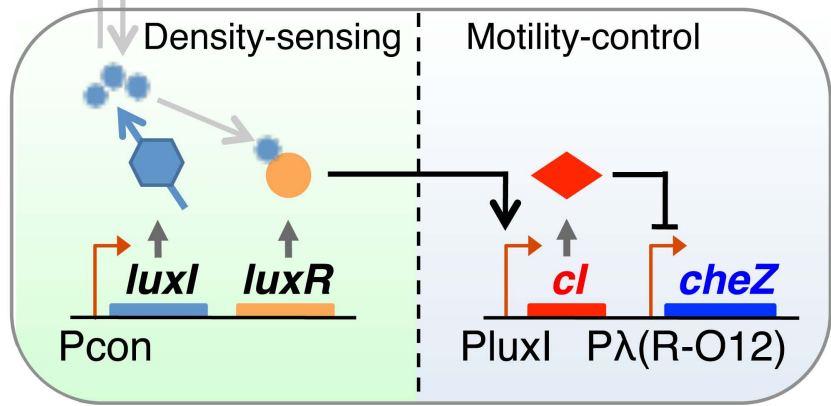

C

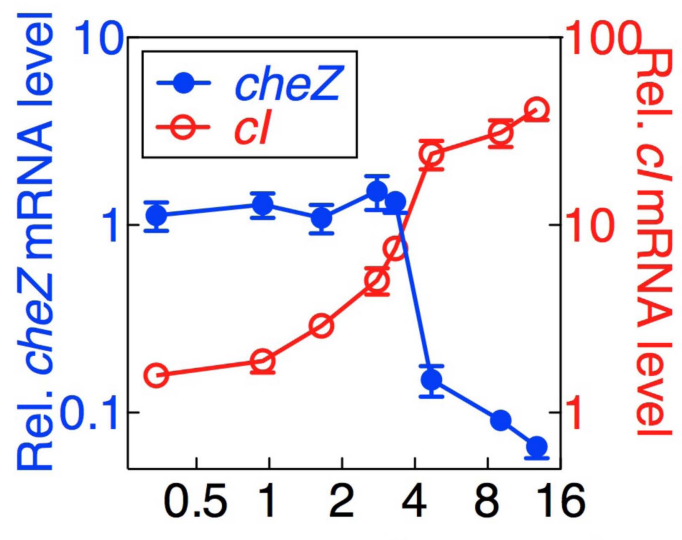

Cell density $\left(10^{8}\right.$ cells $\left.\mathrm{ml}^{-1}\right)$
D

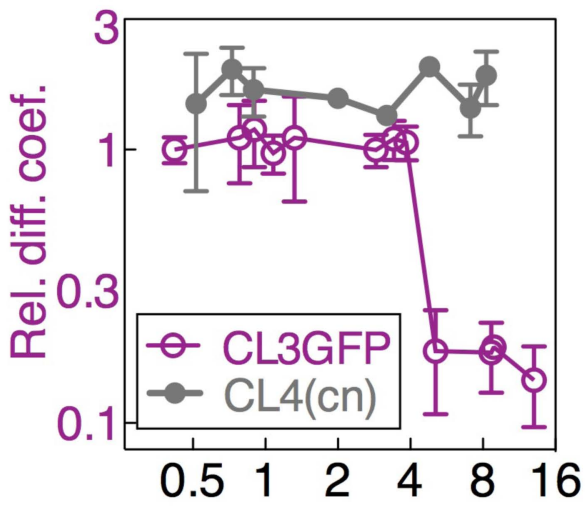

Cell density $\left(10^{8}\right.$ cells $\left.\mathrm{ml}^{-1}\right)$

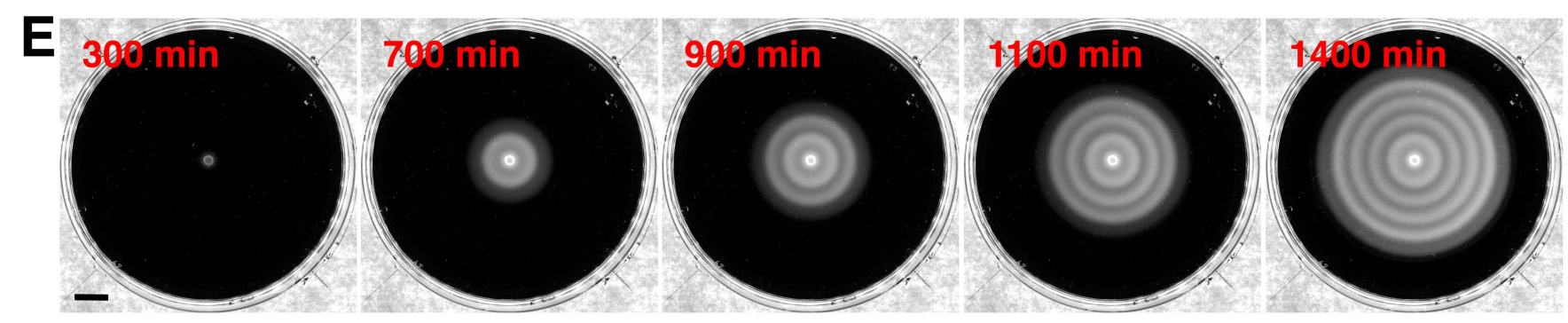

$\mathbf{F}$
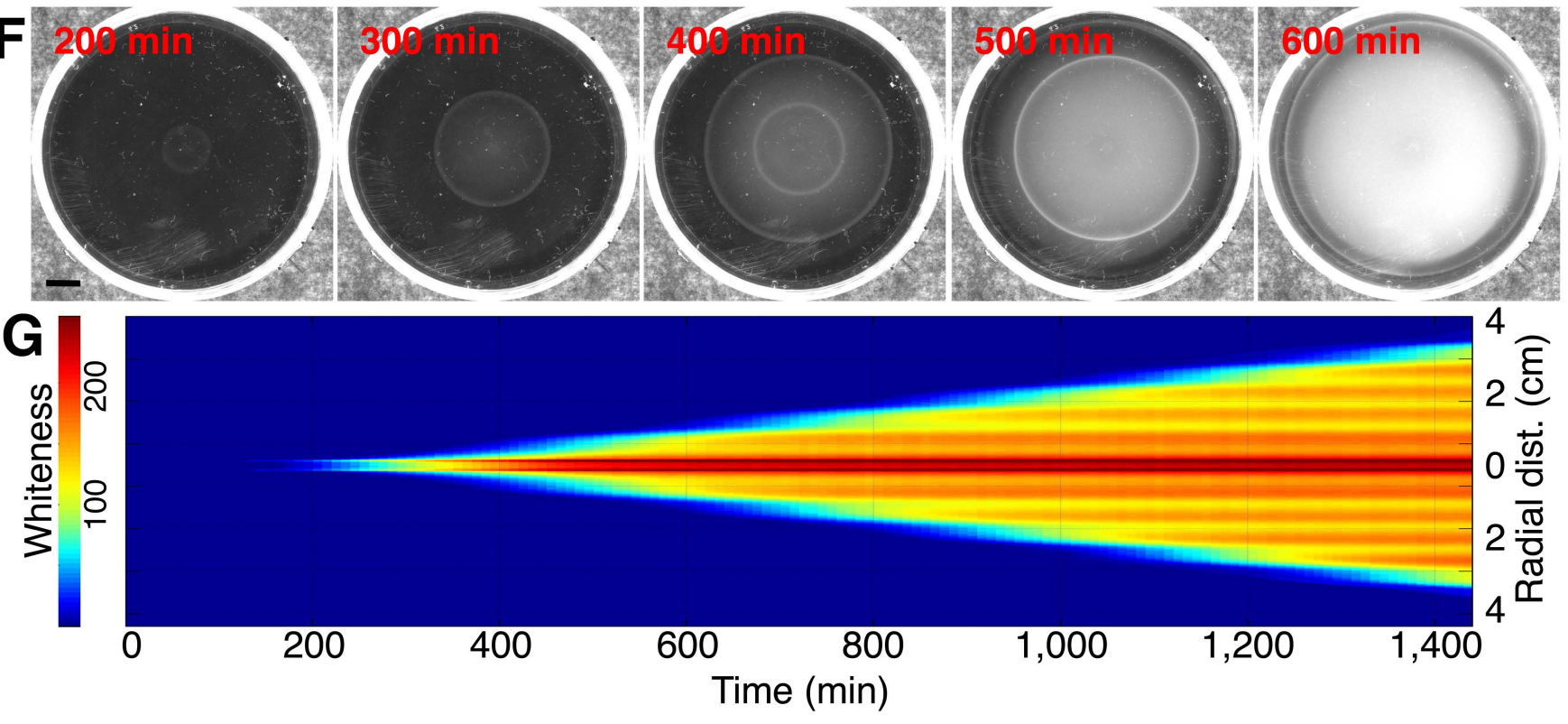
A

$\frac{\partial \rho}{\partial t}=\nabla^{2}[\mu(h) \rho]+\frac{\gamma n^{2} \rho}{n^{2}+K_{n}}$

$\frac{\partial h}{\partial t}=D_{h} \nabla^{2} h+\alpha \rho-\beta h$

$\frac{\partial n}{\partial t}=D_{n} \nabla^{2} n-\frac{k_{n} \gamma n^{2} \rho}{n^{2}+K_{n}}$

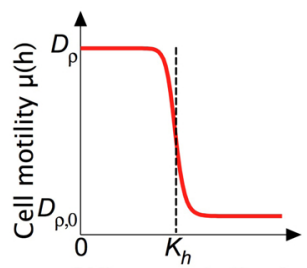

AHL concentration $h$
B - rel. cell density - rel. AHL conc.

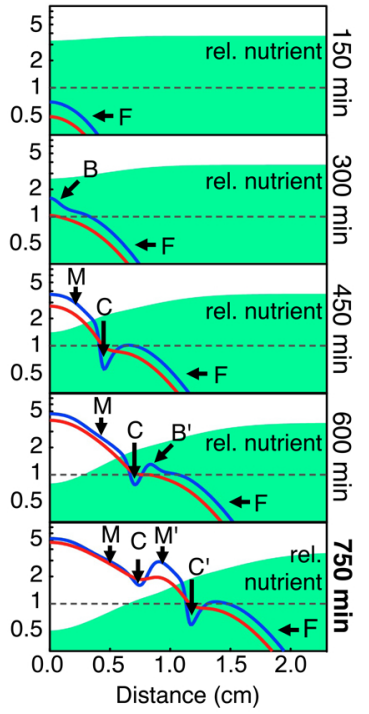


A
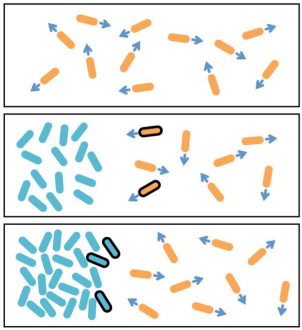

Brightfield Fluorescence

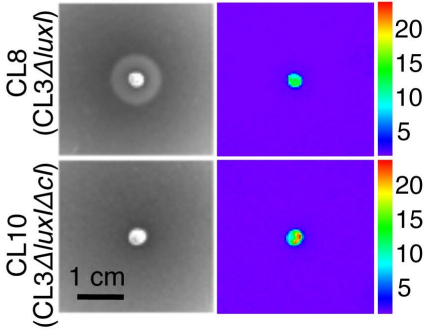


A

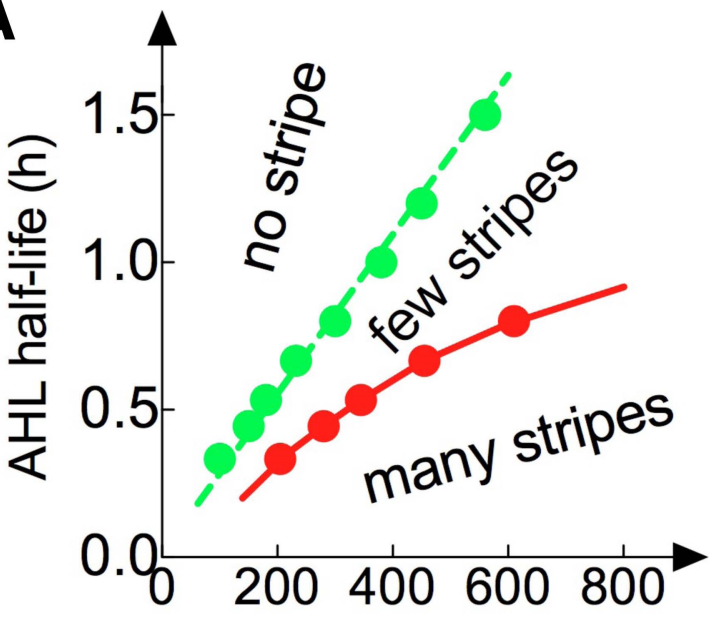

Maximum motility $D_{\rho}\left(\mu \mathrm{m}^{2} \mathrm{~s}^{-1}\right)$

B

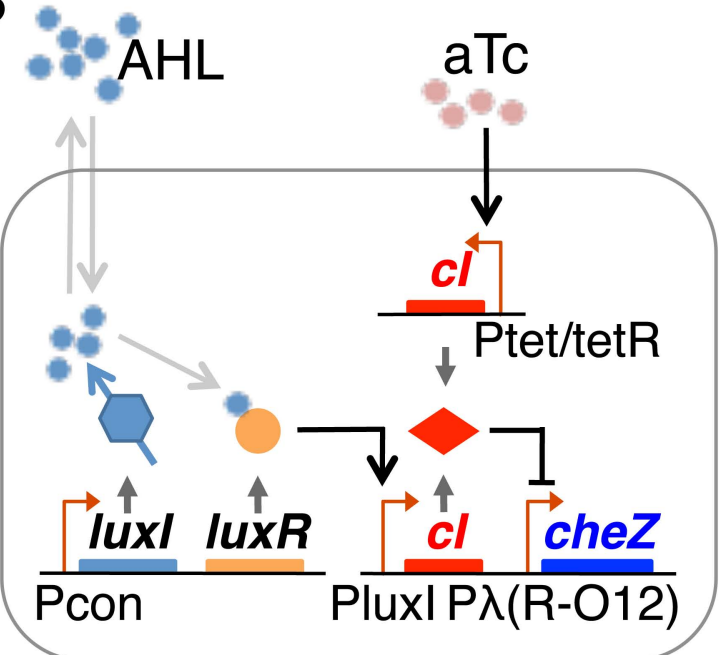

C

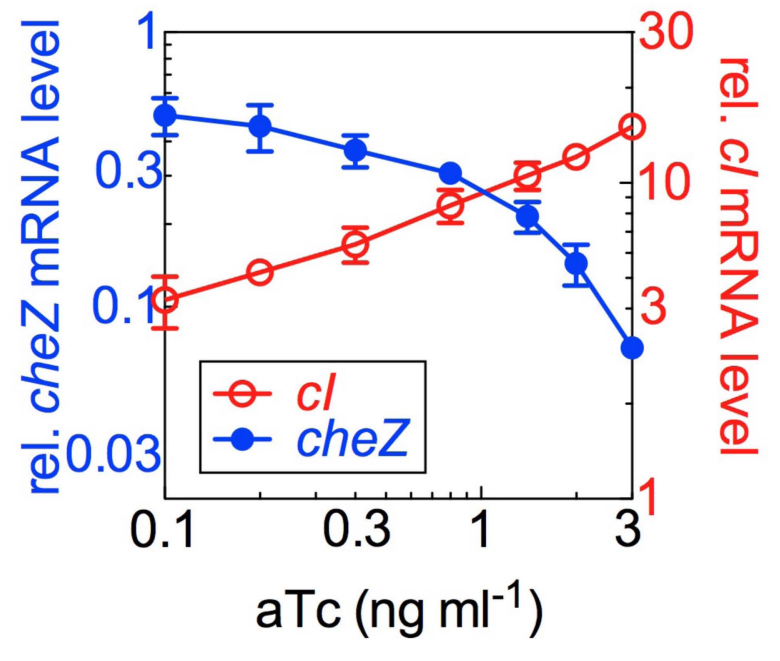

D

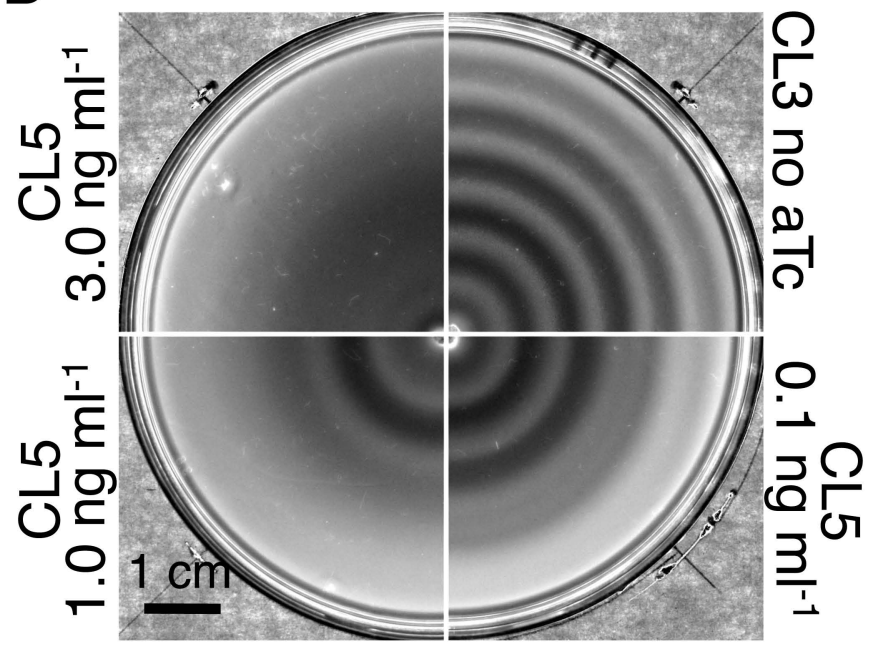




\section{SUPPORTING ONLINE MEATERIAL}

\section{CONTENTS}

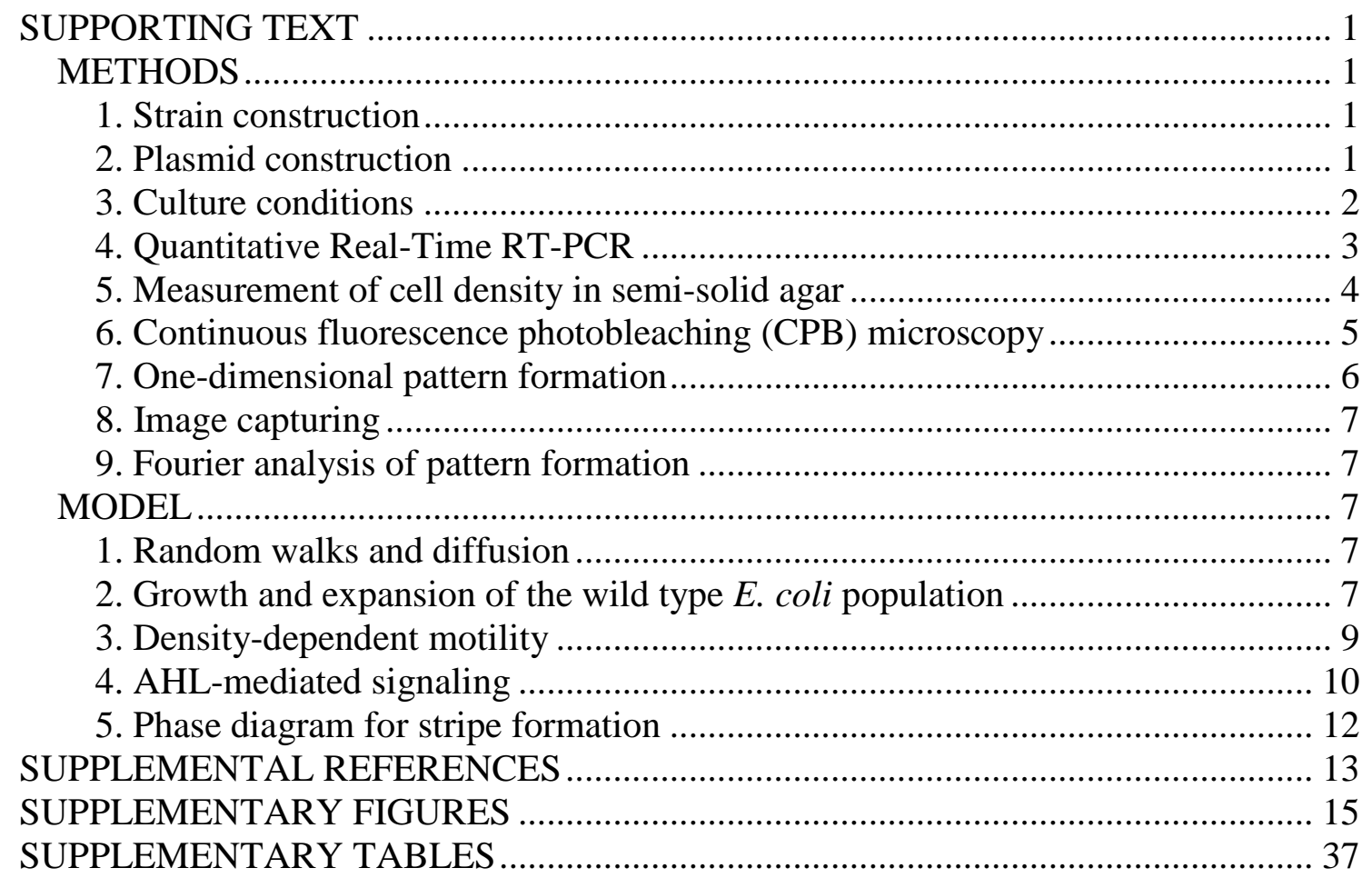




\section{SUPPORTING TEXT}

\section{METHODS}

\section{Strain construction}

Strains and plasmids used in this study are listed in Table S1. Oligonucleotides used are listed in Table S2.

Strain CL-M is a motile E. coli K12 AMB1655 strain, and strain CL-IM is a flagella-lacking mutant of E. coli K12 MG1655 strain. Both are kindly provided by Prof. Antoine Danchin (AMAbiotics, France). To rewire the motility regulation, full-length cheZ in CL-M was deleted "seamlessly" using a recombineering protocol (33) with the aid of pSIM6 (34). Briefly, loxP flanked chloramphenicol resistance cassette was PCR amplified from pEGFP-loxP-CmR-loxP (33) with primers cheZ-del-f and cheZ-del-r (Table S2), each composed of a 50-bp sequence at the 5'-end homologous to the cheZ gene region. The PCR products were treated with DpnI (NEB), gel purified using a QIAquick Gel Extraction Kit (Qiagen), and then electroporated into CL-M cells containing pSIM6 (34) that encodes lambda-Red proteins. $\mathrm{Cm}^{\mathrm{r}}$ colonies were verified for replacement of cheZ with the loxP-CmR-loxP cassette using colony PCR with primers cheZ-del-conf-f and cheZ-del-conf-r, and followed by direct sequencing. Removal of the chloramphenicol gene insertion was mediated by p705Cre as described previously (33). pSIM6 and p705Cre were removed from the host by growing the mutants overnight at $37^{\circ} \mathrm{C}$, since they both carried temperature-sensitive origin of replication, pSC101. Furthermore, we made seamless deletion of the lac operon (including lacI) using the same recombineering protocol, to avoid possible interference with the synthetic $P_{\text {lac/ara-1 }}$ promoter used to drive the expression of $l u x R I$ in pLuxRI2 and its derivatives. The final mutant, designated as CL1, contains seamless deletion of cheZ and the lac operon. Similarly, strain CL1RB was constructed by replacing cheR cheB on the chromosome with a bla gene.

Strain CL2 was constructed via P1 transduction with a recipient strain (CL1) and a donor strain (EQ44) (35), by which the $P_{\mathrm{LTet}-\mathrm{O} 1}$-tetR construct (containing the tet $R$ gene driven by the synthetic, TetR-repressible $P_{\text {LTet-O1 }}$ promoter) and the closely linked bla gene was integrated into the $a t t B$ site. Ampicillin-resistant colonies were verified for

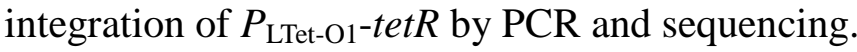

Other strains listed in Table S1 were made by transforming appropriate plasmids into strain CL-M, CL-IM, CL1, CL2, or CL1RB.

\section{Plasmid construction}

pCIcheZ (Fig. S9A) was constructed in two steps. First, luxI in pLD (9) was replaced by the cheZ structure gene amplified by PCR from the genomic DNA of strain CL-M. The ribosomal binding site (RBS) of cheZ was kept the same as that of luxI in pLD. Second, the resultant plasmid was linearized by inverse PCR with primers pLD-rot-f-xba and pLD-rot-r-cla, creating $\mathrm{XbaI}$ and ClaI sites, and ligation with PCR-amplified $P_{\text {luxI }}-C I$ from pLD. pLuxRI/CI (Fig. S9B) was made by inserting a fragment containing $P_{\text {tet }}{ }^{-} \mathrm{CI}$ from restriction-digested pCRTcI into pLuxRI2 (36). pCRTcI was made by inserting PCR-amplified $c I$ from pCIcheZ with ScaI and NotI restriction sites into pCRT-1 (37). 
To construct pLuxRI/GFP, superfolder-gfp (38) was PCR-amplified from BBa_I746916 (Biobrick) with primers gfp-r-sac and gfp-f-sac, composed of a 35-bp sequence of a synthetic constitutive promoter (Biobrick, BBa_J23100) at the 5' end. The fragment was then inserted into pLuxRI2 at the SacI site. The colonies with the correct orientation were confirmed by PCR with primers lac-del-conf-f and gfp-conf-r. To make pCIcheZ $\Delta$ cheZ, pCIcheZ was digested with NheI and EcoRV. The resultant fragment was blunted by T4 DNA polymerase (NEB), then self-ligated. pCIcheZ $\Delta$ cI was made by self-ligating inverse-PCR-amplified fragments from pCIcheZ to exclude the coding regions of $c I$. pLuxR was made by digesting pLuxRI2 with BamHI and NotI to delete luxI, blunting and self-ligating the resultant fragment. Similarly, pLuxI was made by digesting pLuxRI2 with EcoRI and BamHI. pCIcheZ16S was constructed by inserting PCR-amplified 16S rRNA gene with ClaI and XhoI sites into pCIcheZ. All the constructs were confirmed by DNA sequencing.

Since CL1 and its derivatives are lacI-null and also arabinose is never added to the medium, luxR and luxI in pLuxRI2 and its derivatives may be regarded as constitutively expressed. For simplicity, we refer to the promoter of $\operatorname{luxR}$ and $\operatorname{luxI}$ as Pcon.

\section{Culture conditions}

Unless otherwise stated, the following conditions were used throughout this study. Plasmids were maintained with $50 \mu \mathrm{g} \mathrm{ml}^{-1}$ Kanamycin and $25 \mu \mathrm{g} \mathrm{ml}^{-1}$ Chloramphenicol. It had been shown that both AHL degradation rate (39) and E. coli cell motility (40) were influenced by $\mathrm{pH}$ change in media. To minimize the effects of $\mathrm{pH}$, for bulk culture, we used Luria-Bertani (LB) medium containing $2.5 \mathrm{~g}$ Yeast extract, $5 \mathrm{~g}$ Bacto Tryptone, $5 \mathrm{~g} \mathrm{NaCl}$ per liter, buffered by $0.1 \mathrm{M}$ HEPES ( $\mathrm{pH}$ 8.0). The $\mathrm{pH}$ variation was less than 0.3 (measured with PB-10 pH Meter, Sartorius). To prepare semi-solid agar, 10-ml of the above medium containing $0.25 \%$ agar (Difco, Bacto agar) was poured into a Petri dish (8.5-cm internal diameter, Sterilin), and allowed to harden at room temperature for $90 \mathrm{~min}$. For the experiments with varying degree of $c I$ expression, various concentrations of the inducer anhydrotetracycline (aTc, Clontech) were added to the same media before the agar hardened. When rich defined medium (Teknova, Hollister, CA) was utilized, 0.1 M HEPES ( $\mathrm{pH} 8.0$ ) was also added and $0.5 \%(\mathrm{w} / \mathrm{v})$ glycerol was used as the carbon source. Unless otherwise stated, all other reagents were from Sigma. All experiments were carried out at $37^{\circ} \mathrm{C}$.

A single frozen glycerol stock was used throughout this study for each bacterial strain. For each experiment, cells were streaked onto LB agar with appropriate antibiotics from corresponding frozen glycerol stock. 3 to 5 colonies were randomly picked for the following experiments. Medium was pre-warmed prior to use. Before each experiment, cells underwent seed culture and preculture, and then went into experimental culture. Strains were first inoculated from fresh single colonies and cultured in 14-ml tubes (Falcon) with vigorous shaking (seed culture), followed by a round of preculture growth in the same medium (until the mid-exponential phase). After that, cells were subcultured (1:200 dilution) into fresh pre-warmed medium with appropriate antibiotics and aTc, when applicable. For gene expression experiments, samples were taken at different time points for measurements of $\mathrm{OD}_{600}$ (measured with Cary Bio 300 UV-Vis Spectrophotometer, Varian) and RT-qPCR (see Method S4). For pattern formation 
experiments, when $\mathrm{OD}_{600}$ reached 0.1-0.2, a suspension of cells $(2-\mu \mathrm{l})$ was spotted at the center of a pre-warmed semi-solid agar plate. To measure relative diffusion coefficient in semi-solid agar, after preculture growth, cells were diluted 200-fold into the same pre-warmed media containing $0.25 \%$ agar. Cell-agar mixtures were poured into Petri dishes and allowed to harden at room temperature for $90 \mathrm{~min}$, and then moved back into a $37^{\circ} \mathrm{C}$ incubator. After various durations of incubation, plates were taken out for measurements of cell density (see Method S6) and diffusion coefficient (see Method S5) in triplicate. Similarly, for the aggregation test, receiver cells were diluted 10 -fold into a fresh medium containing $0.25 \%$ agar when $\mathrm{OD}_{600}$ reached $0.1-0.2$. Sender cells were grown to the same OD value and concentrated 16-fold, unless otherwise stated. After the cell-agar mixture solidified, $5-\mu 1$ of sender cells was spotted at the center, followed by 12 -h incubation at $37^{\circ} \mathrm{C}$.

\section{Quantitative Real-Time RT-PCR}

Approximately $10^{8}$ cells from each condition were immediately mixed with RNA Bacteria Protect Reagent (Qiagen). Total RNA was extracted using the RNeasy Mini kit (Qiagen) according to the manufacturer's protocol, followed by treatment with Amplification Grade DNase I (Invitrogen). RNA quality was checked by electrophoresis on agarose gel and the absence of DNA contamination was confirmed by PCR. RNA yield was estimated using a NanoDrop 1000 spectrophotometer (Thermo Scientific). To reverse transcribe RNA, about $0.4 \mu \mathrm{g}$ RNA, $200 \mathrm{ng}$ random primer (Invitrogen), 0.5 $\mathrm{mM}$ dNTP Mix, and up to $13 \mu \mathrm{l}$ RNase-free distilled water was mixed and heated to $65{ }^{\circ} \mathrm{C}$ for $5 \mathrm{~min}$ and chilled in ice for $1 \mathrm{~min}$. Then $10 \mu \mathrm{M}$ DTT, 10 unit SuperScript ${ }^{\mathrm{TM}}$ III Reverse Transcriptase (Invitrogen) and $1 \mu 1$ RNaseOUT $^{\mathrm{TM}}$ Recombinant RNase Inhibitor (Invitrogen) were added. The reaction was incubated for $5 \mathrm{~min}$ at $25{ }^{\circ} \mathrm{C}, 60$ $\min$ at $50{ }^{\circ} \mathrm{C}$, and $15 \mathrm{~min}$ at $80{ }^{\circ} \mathrm{C}$. Reactions without reverse transcriptase were conducted as controls for the following qPCR reactions. The cDNA samples were diluted 1:40 with PCR grade water and stored at $-20^{\circ} \mathrm{C}$ until use. Power SYBR Green PCR Master Mix (ABI) was used for qPCR amplification of the amplified cDNA. $1 \mu 1$ of the 1:10 cDNA dilution, $200 \mathrm{nM}$ of forward and reverse qPCR primers, $10 \mu \mathrm{l}$ of SYBR Green PCR Master Mix, and up to $20 \mu \mathrm{l}$ of PCR grade water were mixed in a well of a MicroAmp Fast Optical 96-well reaction plate (ABI). The non-template control (NTC), containing sterile water instead of cDNA template, was included during each qPCR experiment to check the purity of the reagents. Each reaction was performed in triplicate. The qPCR reactions were performed using StepOnePlus ${ }^{\mathrm{TM}}$ Real-time PCR system (ABI) with the following programme: $2 \mathrm{~min}$ at $50{ }^{\circ} \mathrm{C}, 10 \mathrm{~min}$ at $95{ }^{\circ} \mathrm{C}$, and 40 cycles of denaturation $\left(10 \mathrm{~s}, 95^{\circ} \mathrm{C}\right)$, annealing and elongation $\left(30 \mathrm{~s}, 60^{\circ} \mathrm{C}\right)$. Data were acquired at the end of the elongation step. The specificities of accumulated products were verified by melting-curve analysis. A melting curve was run at the end of the 80 cycles to test for the presence of a unique PCR reaction. The qPCR products were checked by electrophoresis on $2 \%$ agarose gels. The primers for real-time PCR were designed with the Primer premier 5.0 software package (http://www.premierbiosoft.com/) and synthesized by Invitrogen (Table S2). DNA gyrase subunit B (gyrB), D-1-deoxyxylulose 5-phosphate synthase (dxs) and 16S ribosomal RNA (16S rRNA) were tested as reference genes. They were analyzed in various cell density conditions $\left(\mathrm{OD}_{600}=0.05-1.2\right)$ and aTc concentrations $\left(0-3 \mathrm{ng} \mathrm{ml}^{-1}\right)$. 
All of them showed comparative low variation in various conditions. Unless otherwise stated, 16S rRNA was used to normalize the abundance of various genes of interest. The relative fold change in mRNA quantity was calculated for each gene using the relative standard curve method (41). To construct the standard curves for 16S rRNA, $c I$, and cheZ, pCIcheZ16S (see Method S2) was 10-fold serially diluted, ranging from $10^{-5}$ to 10 ng $\mu l^{-1}$. For each RNA preparation, at least three independent real-time PCR measurements were performed.

\section{Measurement of cell density in semi-solid agar}

Following the same principle used in the regular spectrophotometer, we determined the cell density by measuring light transmittance of the cell-containing agar plate. Specifically, we positioned a parallel laser beam from $2 \mathrm{~mW} / 650 \mathrm{~nm}$ laser diode (DA650-2-3, Huanic Co., Xi' an, China) perpendicularly to the agar plates and collected the light with a convex lens $(f=75 \mathrm{~mm})$ with an amplified Si photodetector (PDA36A, Thorlabs, NJ, USA). To ensure spatial resolution, the laser beam was passed through a $300-\mu \mathrm{m}$ pinhole. The Petri dish was placed on a motorized stage controlled by a computer running a customized LabVIEW program (National Instruments, Austin, TX, USA) to facilitate spatial and temporal measurements. The whole setup was kept in a warm room $\left(37^{\circ} \mathrm{C}\right)$ throughout the experiment. See Fig. S10.

The 10-ml $0.25 \%$ agar in Petri dish usually forms an inverse tapered shape with the lowest depth at the center. To avoid such systematic error on the optical path length, we measured the location-dependent transmittances for known standard cell densities. Hence we established a series of standard curves for any given radical positions. The agar plates with standard cell densities were generated with cells collected at the mid-exponential phase $\left(\mathrm{OD}_{600}=0.1-0.2\right)$. The cell growth was stopped by washing twice with nutrient-depleted LB (42), and concentrated to $9.6 \times 10^{9}$ cells ml m $^{-1}$. Subsequently, serial dilutions were carried out. For each cell density, $15 \mathrm{ml}$ of cells was vigorously mixed with an equal volume of pre-warmed nutrient-depleted medium containing $0.5 \%$ agar and poured into 3 Petri dishes with $10 \mathrm{ml}$ each. All dishes were allowed to harden at room temperature for $90 \mathrm{~min}$. The final cell densities ranged from 0.03 to $9.6 \times 10^{9}$ cells $\mathrm{ml}^{-1}$. Dishes were moved into a warm room $\left(37^{\circ} \mathrm{C}\right)$ and placed on the motorized stage, and light transmittance measurements were taken every $0.2-\mathrm{mm}$ along the line across the center of the dish. The output laser intensities were digitized at $10 \mathrm{KHz}$ (12 bit) using a DAQ device from National Instruments (NI USB-6009, National Instruments, Austin, TX, USA). The average of 1,000 data points was used to reduce the noise in the measurements at each position. The constant input laser intensity was ensured throughout the experiments with a regulated $5 \mathrm{~V}$ power supply, verified using the photodetector. At each position along the radius, the ratio of the output intensities to the input intensities (measured using a blank agar plate), namely the transmittances $(T)$, were plotted against the known cell densities $\rho$ for 21 different seeding densities (an example curve is shown in Fig. S11A). In total we generated 376 position-dependent density-transmittance standard curves (using a step size of $0.2 \mathrm{~mm}$ for a scanning range of $75 \mathrm{~mm}$ ) and fitted the results to an exponential function for each position $x$ : 


$$
T(x)=A(x) \exp [B(x) \cdot \rho]
$$

where $A(x)$ and $B(x)$ are the position-dependent fitting parameters (Fig. S11B). From these experimentally determined standard curves, we could invert them to compute the real cell density profile $\rho(x)$ from the measured transmittance profile $T(x)$. In subsequent experiments, we scanned the transmittances across the Petri dish for every experiment sample, and computed the spatial cell density profiles. The temporal density profiles were obtained by scanning the agar dish every $10 \mathrm{~min}$ with the help of a customized LabVIEW program.

\section{Continuous fluorescence photobleaching (CPB) microscopy}

A crucial feature needed to characterize the engineered strain is the density dependence of cell motility in semi-solid agar. Microscopy-based single-cell tracing methods are technically challenging to implement at high cell densities where the cells frequently run into each other, and in thick agar where the cells move in-or-out of focus in the $z$-direction. In addition, a large number of single-cell tracing experiments need to be carried out over long time periods to obtain good statistics. Since the swim-and-tumble motion of E. coli cells could be described macroscopically as a diffusive process (43), we aimed to directly quantify the density dependence of diffusion with a population of E. coli cells.

Here, we modified a continuous fluorescence photobleaching (CPB) method (44) to quantify the random motion of $E$. coli cells in semi-solid agar. This method utilized a fine laser beam to photobleach green fluorescent proteins (GFP) expressed by E. coli cells, and deduced the diffusion coefficient from the decay time of photobleaching. This is based on the fact that the diffusion of E. coli cells in and out of the photobleaching region affects the decay of fluorescent intensity. The faster the diffusion, the faster the unbleached GFP-carrying E coli cells refreshed the bleaching region, which lead to slower decay of the total fluorescent intensity observed.

A fluorescence photobleaching microscopy was set up using a Nikon inverted microscope (Eclipse TE2000-E, Nikon Instruments, Kawasaki, Kanagawa, Japan) as illustrated in Fig. S12. An environmental chamber was built maintain the temperature inside at $37{ }^{\circ} \mathrm{C}$ throughout the experiments. A laser beam from a 473-nm solid-state laser (MBL-III-473/30mW, Changchun New Industries Co., Changchun, China) illuminated through the semi-solid agar from the top to the bottom, to excite and bleach fluorescent proteins in cells. The emitted fluorescence signal was collected with a $10 \times$ objective (Nikon Plain Fluor 10×/NA0.3) and filtered through a narrow green bandpass filter (center wavelength=515 nm, FWHM=10 nm, Omega Optical, Brattleboro, VT, USA). The fluorescent image was recorded every second with a CMOS camera (DCC1545M, Thorlabs, NJ, USA).

In our experiments, CL3GFP, CL4 (CL-M expressing GFP), or CL14 (CL-IM expressing GFP) cells were uniformly mixed in $0.25 \%$ agar at low cell density and cultured at $37^{\circ} \mathrm{C}$ (Method S3). At each time point, we measured the average fluorescent intensity in the photobleaching region for each fluorescence image, and obtained a time series of fluorescent intensity for each location of each sample, $i(t)$. To control for variations in cell densities and obtain specific GFP signals, we defined a GFP-specific 
relative fluorescent intensity $(I(t))$ as

$$
I(t)=\frac{i(t)-i_{B}(t)}{i(0)-i_{B}(0)}
$$

where $i(t)$ is the average of the fluorescent intensity, and $i(0)$ is the value at $t=0$. Agar plates with similar cell densities but no GFP expression were measured in parallel as $i_{B}(t)$. The background signal $i_{B}(t)$ was constituted of autofluorescence from LB medium, agar and cells. The latter had minimal contribution. Examples of the continuous fluorescence photobleaching curve with various fitted values of cell diffusion coefficients are shown in Fig. S13A.

We used strain CL14, a flagella-lacking mutant, as an immobile reference $(D=0)$ throughout the study. It provided us with a pure GFP photobleaching curve $I(t)=f(t)$ (Fig. S13A). The curve is best described by a double-exponential decay, with the half-lives of $\sim 20 \mathrm{~s}$ and $\sim 170 \mathrm{~s}$ for each component, respectively (Fig. S13A). This two-component photobleaching has been observed in a number of fluorescent proteins (45), especially for the GFP variant (superfolder GFP) we used (38). As we kept the laser power constant, the two decay constants remained the same throughout all our experiments. For the mobile strains, the decays were, as we expected, slower (Fig. S13A). Then we adapted a single-cell simulation-based process to derive the diffusion coefficients from experiments (Fig. S13A). For a given diffusion coefficient, we performed computer simulations to mimic the Brownian motion of many cells, and assigned the time-dependent fluorescent intensity for each cell based on the accumulated time that it was located in the bleaching zone and the two decay constants. Both the decay constants and the size of the bleaching zone used in the simulation were determined experimentally (Fig. S13A). With the trajectories of fluorescent intensities of many cells, we can compute the total fluorescent intensity in the photobleaching zone. We plotted the relation between diffusion coefficients and the averages of relative fluorescent intensities from 175 to $180 \mathrm{~s}$ (Fig. S13B). This was used as the standard curve to compute the diffusion coefficients from experimentally measured bleaching curves. However, the exact value of diffusion coefficient depends on evaluation of the sizes of the bleaching zone $\left(R^{2}\right)$. This size is hard to determine due to the lacking of well-defined boundary, as laser beam exhibits a cross-sectional Gaussian intensity distribution. Therefore, we reported relative diffusion coefficients in Fig. 1D.

To measure the relative fluorescent intensity curves for different cell densities in semi-solid agar, cell-containing semi-solid agar plates were prepared as described in Method S3. Every 20-30 min, we took two plates out to measure the cell density (see Method S5), followed by the measurement of the fluorescent intensity curves for $200 \mathrm{~s}$. Three positions were randomly measured for each plate. The exposure time was adjusted between 0.05 and $1 \mathrm{~s}$ to match the dynamic range of the camera. The boundary for the photobleaching region was computed using the segmentation algorithm developed by Otsu et al (46).

\section{One-dimensional pattern formation}

We made rectangular plastic plates of two sizes: $3 \mathrm{~cm}$ by $7 \mathrm{~cm}$ and $6 \mathrm{~cm}$ by $10 \mathrm{~cm}$. 3and $10-\mathrm{ml}$ of $0.25 \%$ agar was added to the small and large size plates, respectively. 
After the agar hardened, cells were horizontally inoculated as a line using a sterile coverslip (47) and incubated overnight at $37^{\circ} \mathrm{C}$.

\section{Image capturing}

The pseudo darkfield images of agar plates were taken using a Canon EOS 450D digital camera with a Canon EF $50 \mathrm{~mm}$ f/1.8 II lens and an exposure setting of f5.6/0.6s/ISO200. The agar plates were illuminated by a circular white LED array with the diameter of $30 \mathrm{~cm}$ and $10 \mathrm{~cm}$ bellow, similar to what was described by Budrene and Berg(48). The time-lapse photographs were acquired with the aid of a timer shutter release controller (TC-80N3a, YongNuo, HK, China). Fluorescence images of the agar plates were captured using a Xenogen IVIS 100 imaging system (Caliper LifeSciences, Hopkinton, MA, USA) with the exposure time of $30 \mathrm{~s}$.

\section{Fourier analysis of pattern formation}

The time-lapse imaging for 1-D stripe formation was used for Fourier analysis. At each time point, a 1-D intensity profile was computed perpendicular to the stripes. These 1-D profiles were padded with mean intensities to 1024 pixels and applied with a Hamming window. A standard MATLAB (Mathworks, Natick, MA, USA) built-in Fast Fourier Transform (FFT) function was used to compute the mean square power spectra from the 1-D intensity profiles.

\section{MODEL}

\section{Random walks and diffusion}

The run-and-tumble motion of E. coli cells has been studied for decades (43). On the molecular level, E. coli cells switch between run-and-tumble states of motion by controlling the direction of flagella rotation. When the flagella rotate in a counterclockwise fashion, the bacterium swims forward, otherwise it tumbles. The typical swimming speed of wild type E. coli cells in liquid culture is about $10 \sim 20 \mu \mathrm{m} \mathrm{s}^{-1}$. Cells tumble once every $0.5 \sim 1 \mathrm{~s}$ (43). Macroscopically, the continuous run-and-tumble motion of E. coli cells can be described by random walks with step size of $10 \sim 20 \mu \mathrm{m}$, changing direction completely every $0.5 \sim 1 \mathrm{~s}$. Such random walks can be described by a diffusion equation, particularly at a population level. E. coli K12 cell motion in liquid culture are described with a diffusion coefficient ranging from 200 to $1,000 \mu \mathrm{m}^{2} \mathrm{~s}^{-1}$ (49-51).

\section{Growth and expansion of the wild type E. coli population}

For wild type (WT) E. coli grown in the semi-solid agar plate (0.1-0.3\%), which allows the cells to swim, the spatiotemporal dynamics is governed by two ingredients: cell motility and cell growth. Modeling cell motility by diffusion and cell growth by logistic growth (52) in the simplest case, we have 


$$
\frac{\partial \rho}{\partial t}=D_{\rho} \nabla^{2} \rho+\gamma \rho \cdot\left(1-\rho / \rho_{s}\right)
$$

for the dynamics of cell density $\rho$, with $D_{\rho}$ being the diffusion coefficient, $\gamma$ being the growth rate, and $\rho_{s}$ being the saturated cell density (also known as the "carrying capacity"). [Note that while the term $-\rho / \rho_{s}$ in the above equation may be interpreted mathematically as due to cell death (21), bacterial death is negligible over the time scale of our experiments and this term is used here as a mathematical convenient way to describe the effect of nutrient depletion. All the results presented in this work are generated by explicitly including the nutrient field as described below.]

Eq. [S.3] is known as the Fisher's equation ${ }^{21}$. It admits a propagating front solution for localized initial cell populations. In one dimension, the propagation speed is given by $2 \sqrt{D_{\rho} \gamma}(52,53)$. In two dimensions, the equation for a radial symmetric profile can be written as

$$
\frac{\partial \rho}{\partial t}=D_{\rho}\left(\frac{\partial^{2} \rho}{\partial r^{2}}+\frac{1}{r} \frac{\partial \rho}{\partial r}\right)+\gamma \rho \cdot\left(1-\rho / \rho_{s}\right)
$$

in polar coordinate with radial distance $r$. The front propagation speed of the traveling wave approaches that of the 1D case when $r$ is large (53).

Using typical parameters relevant to the WT strain under our experimental conditions, $D_{\rho}=200-1,000 \mu^{2} \mathrm{~s}^{-1}$, (49-51) $\rho_{\mathrm{s}}=15 \times 10^{8}$ cells ml ${ }^{-1}$ (corresponding to $\mathrm{OD}_{600^{\sim}} 2.5$ ), $\gamma=1.4 \mathrm{~h}^{-1}$ (doubling time of $\sim 30 \mathrm{~min}$ ) as summarized in Table S3, the propagating speed is not larger than $2 \mathrm{~mm} \mathrm{~h}^{-1}$ (see Fig. S15A), which is significantly below the observed speed of $5 \mathrm{~mm} \mathrm{~h}^{-1}$ (i.e. $1.4 \mu \mathrm{m} \mathrm{s}^{-1}$ ) (Fig. S15C). One likely reason for this discrepancy is bacterial chemotaxis, which is believed to be the cause for the bright rings leading the propagating front (known as the Adler's ring) (54); see Fig. S15C. There are a number of models to describe the chemotaxis effect. Here we follow the model by Woodward et al (55), in which a drift term (in the direction of the gradient of the attractant $a$ ) is added to Fisher's equation, and growth is coupled directly to the nutrient concentration $(n)$, with $a$ and $n$ both consumed by the cells:

$$
\begin{aligned}
& \frac{\partial \rho}{\partial t}=D_{\rho} \nabla^{2} \rho-\nabla \cdot\left(\frac{D_{\rho} K_{C_{1}} \rho}{\left(K_{C_{2}}+a\right)^{2}} \nabla a\right)+\frac{\gamma n^{2} \rho}{n^{2}+K_{n}^{2}} \\
& \frac{\partial a}{\partial t}=D_{a} \nabla^{2} a-\frac{k_{a} \gamma a \rho}{a+K_{a}} \\
& \frac{\partial n}{\partial t}=D_{n} \nabla^{2} n-\frac{k_{n} \gamma n^{2} \rho}{n^{2}+K_{n}^{2}} .
\end{aligned}
$$

Here, the strength of the chemotaxis is taken to be proportional to the cell diffusion coefficient $D_{\rho} . \quad K_{C_{1}}$ and $K_{C_{2}}$ are two additional parameters associated with chemotaxis. The consumption rates of the chemoattractant $a$ and nutrient $n$ are assumed to be proportional to the growth rate $\gamma \rho$ with proportionality constants $k_{a}$ and $k_{n}$ respectively. The parameters $K_{a}$ and $K_{n}$ represent the saturation levels of consumption of $a$ and $n$, respectively. 
Eqs.[S.5] -[S.7] again generate a propagating front in one- and two- dimensions, but this time preceded by a ring (Fig. S15B) analogous to the Adler ring observed in experiment (Fig. S15C). The values of the chemotaxis parameters $K_{C_{1}}$ and $K_{C_{2}}$ were adjusted to reproduce the observed propagation speed (Fig. S15B). Of the remaining parameters, the values of $D_{a}$ and $D_{n}$ were chosen based on the diffusion coefficients of similar small molecules $\left(\sim 800 \mu \mathrm{m}^{2} \mathrm{~s}^{-1}\right)(56,57)$, while $k_{a}$ and $k_{n}$ can be scaled out. $K_{a}$ is unknown, but within a reasonable range does not affect the simulation results much. $K_{n}$ can be estimated by the cell growth curve measured in the agar plate. From our experimental data (Fig. S16), the cell growth curve for strain (CL4) shows nearly exponential growth until the cell density reaches $10^{9}$ cells $\mathrm{ml}^{-1}$. The cell density for the stationary phase is $15 \times 10^{8}$ cells $\mathrm{ml}^{-1}\left(\mathrm{OD}_{600} \sim 2.5\right)$, which can be considered as the initial nutrient level in terms of cell density. So $K_{n}$ is about two thirds of the initial nutrient concentration.

\section{Density-dependent motility}

To understand how the uniform propagating front solution exhibited by wild type $E$. coli cells break into spatially nontrivial structures generated by the engineered strain, we start by including the effect of density-dependent cell motility of the engineered strain into Fisher's equation (Eq. [S.3]). We first consider an isotropic random walk, i.e. without chemotaxis. The diffusion of the bacterial population with diffusion coefficient $D_{\rho}$ may be characterized in the following microscopic model: Over some small time internal $\Delta t$, most cells are localized within a spatial region of linear dimension $\Delta x$ and only a small fraction $D_{\rho} \cdot \Delta t /(\Delta x)^{2}$ of randomly chosen cells leave the region (in random directions) to neighboring regions. Density-dependence may be incorporated by multiplying $D_{\rho}$ by a density-dependent function $\varepsilon(\rho)$. Over a region where density varies in space, the macroscopic dynamics of the population is generally not described by diffusion (53), and the following equation of motion can be derived:

$$
\frac{\partial \rho}{\partial t}=D_{\rho} \nabla^{2}(\varepsilon(\rho) \rho)+\gamma \rho \cdot\left(1-\rho / \rho_{s}\right)
$$

More generally, the spatial coupling term proportional to $D_{\rho}$ is given by a mixture of two terms, $\theta \nabla^{2}(\varepsilon(\rho) \rho)+(1-\theta) \nabla \cdot(\rho \nabla \varepsilon(\rho))$, with the value of the parameter $\theta$ being dependent on the details of the microscopic dynamics; e.g., $\theta=1$ for discrete-time (Ito) dynamics and $\theta=1 / 2$ for continuous-time (Stratanovich) dynamics (58). Detailed derivation of a related chemotaxis model found a mixture of two terms (24). As we will see, the important term for pattern formation is $\nabla^{2}(\varepsilon(\rho) \rho)$; to minimize the number of parameters, we will take $\theta=1$ and only keep track of the effect of the $\nabla^{2}(\varepsilon(\rho) \rho)$ term in this study.

Eq. [S.8] is linearly unstable. To see this behavior, consider a small density perturbation of amplitude $\delta \hat{\rho}$ and wavenumber $\vec{k}$ around a time dependent and spatially homogenous solution $\rho_{0}(t)$ that follows logistic growth. If

$$
\begin{gathered}
\rho(\vec{r}, t)=\rho_{0}(t)+\int \delta \hat{\rho}(\vec{k}) e^{i \vec{k} \cdot \vec{r}} \cdot e^{-\lambda t} d k, \text { then } \\
\lambda=D_{\rho} k^{2}\left[\varepsilon^{\prime}(\rho) \rho+\varepsilon(\rho)\right]
\end{gathered}
$$


So if $\varepsilon^{\prime}(\rho) \rho+\varepsilon(\rho)<0$, then Eq. [S.8] would become unstable for all wave numbers, diverging at large $k$. This means Eq. [S.8] is numerically unstable. To avoid the high wavelength instabilities, we introduce the effect of AHL in accordance with our genetic circuit (see next section). In the recent work by Cates et al (21) an ad-hoc $k^{4}$-term was added to Eq. [S.8] to achieve a similar effect.

\section{AHL-mediated signaling}

In the designed genetic circuit (Fig. 1B), a small molecule AHL was used to signal cell density. Increased cell density (implying increased AHL level) reduced the cell's motility abruptly as shown in Fig. 1D. To describe this effect mathematically, we introduce a motility function $\mu(h)$, which depends explicitly on the AHL level $h(x, t)$. At constant cell density $\rho$, macroscopic dynamics is described by diffusion with diffusion coefficient $\mu(h(\rho))$,. Assuming that $h$ and $\rho$ are linearly related, then the measured density-dependent diffusion coefficient (Fig. 1D) suggests an abrupt form of $\mu(h)$, which was modeled with a Hill function,

$$
\mu(h)=\frac{D_{\rho}+D_{\rho, 0}\left(h / K_{h}\right)^{m}}{1+\left(h / K_{h}\right)^{m}}
$$

which has a maximum value of $D_{\rho}$ switching to a minimum value of $D_{\rho, 0}<<D_{\rho}$ at $h \approx K_{h}$ as shown in Fig. 2A.

Including the nonlinear diffusion term as in Eq. [S.8] with $D_{\rho} \varepsilon(\rho)$ replaced by $\mu(h)$ and further including the dynamics of $h$, we have

$$
\begin{aligned}
& \frac{\partial \rho}{\partial t}=\nabla^{2}[\mu(h) \rho]+\gamma \rho \cdot\left(1-\rho / \rho_{s}\right) \\
& \frac{\partial h}{\partial t}=D_{h} \nabla^{2} h+\alpha \rho-\beta h
\end{aligned}
$$

where $\alpha$ and $\beta$ are the synthesis and degradation rate of AHL respectively, and $D_{\rho}$ is the diffusion coefficient of AHL. AHL diffusion is expected to be in the range of small molecule diffusion, 200 1,000 $\mu \mathrm{m}^{2} \mathrm{~s}^{-1}(56,57)$. The range of AHL molecule half-life in a slightly alkaline environment (pH7 9) was estimated to be 10 1,000 min $(39,59)$. The value of $\alpha$ in our experiment is not known and depends on the expression level of AHL-synthesizing LuxI. However, it is only the combination $\alpha / K_{h}$, the rate to synthesize enough AHL to reach the level of $K_{h}$, that affects the outcome. This ratio can be obtained from the density-dependent diffusion coefficient measured in Fig. S1b, for homogenous distribution of cells of different densities mixed in semi-solid agar plate. The data may be fitted by the form of $\mu(h)$ in Eq. [S.10] with $h=\alpha \rho / \beta$. We obtain $\beta K_{h} / \alpha \approx 4 \times 10^{8}$ cells $\mathrm{ml}^{-1}$ together with a lower bound of 10 on the Hill coefficient $m$. In the following calculation, we will use $m=20$.

Eqs. [S.11] and [S.12] were able to initiate patterns. However over time, cell density increased towards $\rho_{s}$ everywhere in space (see Fig. S17A) and no stable pattern could be maintained. Mathematically, patterns could in principle be stabilized at $\rho>\rho_{s}$ provided that $K_{h}$ is large enough so that the instability arising from nonlinear diffusion 
(occurring at $\rho \sim \beta K_{h} / \alpha$ ) balances negative contribution from the logistic growth term (Fig. S17B). This type of patterns could be initiated from small density variation in the initial conditions due to linear instability as discussed by Cates et al (21). However, we believe that they are not applicable to our experimental system for a number of reasons: (i) cell death simulated by the negative growth term does not play a role in our system; and (ii) according to the data $\beta K_{h} / \alpha \approx 0.25 \rho_{s}$, so the instability zone is below $\rho_{s}$ should one wish to cast the numbers within the logistic description (the exact value of $\rho_{s}$ could be affected by the initial nutrient used; in an experimental relevant range, $\rho_{s}$ does not affect the pattern, see Fig. S2H). Under these conditions, our model admits no nontrivial stable states. This is also verified experimentally: starting from initial semi-uniform cell distribution (small fluctuation of densities) on the semi-solid agar plate, no periodic patterns can be formed (Fig. S18).

To circumvent the saturation problem (Fig. S17A), we explicitly include the nutrient field $n(x, t)$ that fuels cell growth and, at the same time is consumed by cell growth. We have

$$
\begin{aligned}
& \frac{\partial \rho}{\partial t}=\nabla^{2}[\mu(h) \rho]+\frac{\gamma n^{2} \rho}{n^{2}+K_{n}^{2}} \\
& \frac{\partial n}{\partial t}=D_{n} \nabla^{2} n-\frac{k_{n} \gamma n^{2} \rho}{n^{2}+K_{n}^{2}}
\end{aligned}
$$

where the parameters and their values are as defined in Eqs. [S.5] and [S.7] and summarized in Table S3. [In the above equations, a Hill coefficient of 2 was used following the model by Woodward et al (55). However, the results on pattern formation can also be obtained using Hill coefficient of 1.]

The system defined by Eqs. [S.12] -[S.14] is capable of generating stable stripes (60). The spatiotemporal dynamics of the stripe formation process in 1D space, shown in Fig. S5, was generated using the parameter values in Table S3, a Gaussian initial cell density profile:

$$
\rho(x, 0)=2 \exp \left(-x^{2} / 4 m^{2}\right) \times 10^{8} \text { cells } \mathrm{ml}^{-1} \text { for } 0 \mathrm{~mm} \leq x \leq 3 \mathrm{~mm}
$$

and $15 \times 10^{8}$ cells $\mathrm{ml}^{-1}$ as the initial nutrient level. We have also performed simulations in 2D. The 2D space model gives the stripe pattern (Fig. S19A) similar to the 1D case, except for minor differences in the amplitude of the periodic structure.

Depending on the detailed microscopic dynamics, in principle there could be also a density-dependent drift term as shown in Eq. [S.16] as discussed above and by Tailleur and Cates (24) (who explicitly coarse-grained the dynamics of a related process where the bacteria's run length is density dependent).

$$
\frac{\partial \rho}{\partial t}=\theta \nabla^{2}[\mu(h) \rho]+(1-\theta) \nabla \cdot(\mu(h) \nabla \rho)+\frac{\gamma n^{2} \rho}{n^{2}+K_{n}^{2}}
$$

We examined its effect (using $\theta=1 / 2$ ), together with Eqs. [S.12] and [S.14] in 1D. The stripes pattern can still be generated as shown in Fig. S20A, although the details appear differently from Fig. S5. 
Finally, the engineered strain is also expected to exhibit chemotaxis as the wild type. As discussed in the previous section, the effect of chemotaxis can be modeled as a drift term towards some chemoattractant gradient. This is implemented by replacing Eq. [S.13] by

$$
\frac{\partial \rho}{\partial t}=\nabla^{2}(\mu(h) \rho)-\nabla \cdot\left(\frac{D_{\rho} K_{C_{1}} \rho}{\left(K_{C_{2}}+a\right)^{2}} \nabla a\right)+\frac{\gamma n^{2} \rho}{n^{2}+K_{n}^{2}}
$$

and further supplementing Eqs. [S.6], [S.12] and [S.14]. Simulation shows that the stripe pattern formed is robust to the presence of chemotaxis; see Fig. S20B.

\section{Phase diagram for stripe formation}

Eqs. [S.12] -[S.14] do not generate periodic stripes for arbitrary combination of parameters. As will be explained below, the behavior of this system is controlled mostly by the two dimensionless combinations of parameters, $D_{\rho} / D_{h}$ and $\gamma / \beta$. By performing the $1 \mathrm{D}$ simulation with varying parameters and a smaller initial cell density:

$$
\rho(x, 0)=2 \exp \left(-x^{2} / 0.1 \mathrm{~mm}^{2}\right) \times 10^{5} \text { cells } \mathrm{ml}^{-1} \text { for } 0 \mathrm{~mm} \leq x \leq 0.2 \mathrm{~mm}
$$

we found that the periodic stripe pattern occurs to the right of the red line in the phase diagram (Fig. 4A). Immediately to the left of the red line, the system can still generate a finite number of stripes if the initial cell density is sufficiently large (e.g. Eq.[S.18]). But in the long-time limit, it eventually turns into a propagating front. No stripes formed far away from the red line.

The separation between "few stripes" and "no stripe", as indicated by the dashed green line, is not crisp and depends on the form of the initial density profile used (here we used the initial condition as Eq.[S.18]). However, we believe the transition across the red line is a real phase transition. Since a well-defined period can be assigned in one phase while no stripes formed in the long-time limit of the other phase, this transition is expected to be a Hopf bifurcation (61).

Similar phase boundary can be found by including the chemotaxis effect Eq. [S.17] with Eqs. [S.6], [S.12] and [S.14]; see Fig. S21. Over the range of the experimental relevant parameters, chemotaxis could either expand or reduce the parameter space for stripe formation. 


\section{SUPPLEMENTAL REFERENCES}

33. R. M. Watt et al., Nucleic Acids Res 35, e37 (2007).

34. S. Datta, N. Costantino, D. L. Court, Gene 379, 109 (2006).

35. $\quad$ S. Klumpp, Z. Zhang, T. Hwa, Cell 139, 1366 (2009).

36. L. You et al., Oncogene 23, 6170 (2004).

37. $\quad$ S. Hooshangi, S. Thiberge, R. Weiss, Proc Natl Acad Sci U S A 102, 3581 (2005).

38. J. D. Pedelacq, S. Cabantous, T. Tran, T. C. Terwilliger, G. S. Waldo, Nat Biotechnol 24, 79 (2006).

39. A. L. Schaefer, B. L. Hanzelka, M. R. Parsek, E. P. Greenberg, Methods Enzymol 305, 288 (2000).

40. L. M. Maurer, E. Yohannes, S. S. Bondurant, M. Radmacher, J. L. Slonczewski, J Bacteriol 187, 304 (2005).

41. C. Lee, S. Lee, S. G. Shin, S. Hwang, Appl Microbiol Biotechnol 78, 371 (2008).

42. G. Sezonov, D. Joseleau-Petit, R. D'Ari, J Bacteriol 189, 8746 (2007).

43. H. C. Berg, E. coli in motion. Biological and medical physics series (Springer, New York, 2004), pp. xi, 133 p., 1 col. plate.

44. K. M. Slade, B. L. Steele, G. J. Pielak, N. L. Thompson, J Phys Chem B 113, 4837 (2009).

45. $\quad$ N. C. Shaner, P. A. Steinbach, R. Y. Tsien, Nat Methods 2, 905 (2005).

46. N. Otsu, Ieee Transactions on Systems Man and Cybernetics 9, 62 (1979).

47. H. S. Girgis, Y. Liu, W. S. Ryu, S. Tavazoie, PLoS Genet 3, 1644 (2007).

48. E. O. Budrene, H. C. Berg, Nature 349, 630 (1991).

49. B. R. Phillips, J. A. Quinn, H. Goldfine, Aiche Journal 40, 334 (1994).

50. P. Lewus, R. M. Ford, Biotechnol Bioeng 75, 292 (2001).

51. R. M. Ford, B. R. Phillips, J. A. Quinn, D. A. Lauffenburger, Biotechnol Bioeng 37, 647 (1991).

52. $\quad$ R. A. Fisher, Ann Eugenics 7, 355 (1937).

53. J. D. Murray, Mathematical biology. Interdisciplinary applied mathematics (Springer, New York, ed. 3rd, 2002).

54. J. Adler, Science 153, 708 (1966).

55. $\quad$ D. E. Woodward et al., Biophys J 68, 2181 (1995).

56. M. Hoistad, K. C. Chen, C. Nicholson, K. Fuxe, J. Kehr, J Neurochem 81, 80 (2002).

57. $\quad$ C. Nicholson, J. M. Phillips, J Physiol 321, 225 (1981).

58. N. G. v. Kampen, Stochastic processes in physics and chemistry. (Elsevier, Amsterdam ; London, ed. 3rd, 2007), pp. xvi, 463 p.

59. E. A. Yates et al., Infect Immun 70, 5635 (2002).

60. Strictly speaking, the pattern formed is not stable at a very long time scale because the diffusion coefficient $D_{\rho, 0}$ at high cell densities is not strictly zero. However, practical time scales reachable by simulations, this effect is not noticeable. For the experiments, we expect the cell's motility to cease completely once the nutrient is depleted. Hence motion over the very long time scale is not experimentally relevant.

61. S. H. Strogatz, Nonlinear dynamics and chaos : with applications to physics, biology, chemistry, and engineering. (Addison-Wesley Pub., Reading, Mass., 1994), pp. xi, 498 p., [4] p. of plates.

62. J. B. Andersen et al., Appl Environ Microbiol 64, 2240 (1998).

63. Y. Zhang, F. Buchholz, J. P. Muyrers, A. F. Stewart, Nat Genet 20, 123 (1998). 


\section{SUPPLEMENTARY FIGURES}

\section{Figure S1}

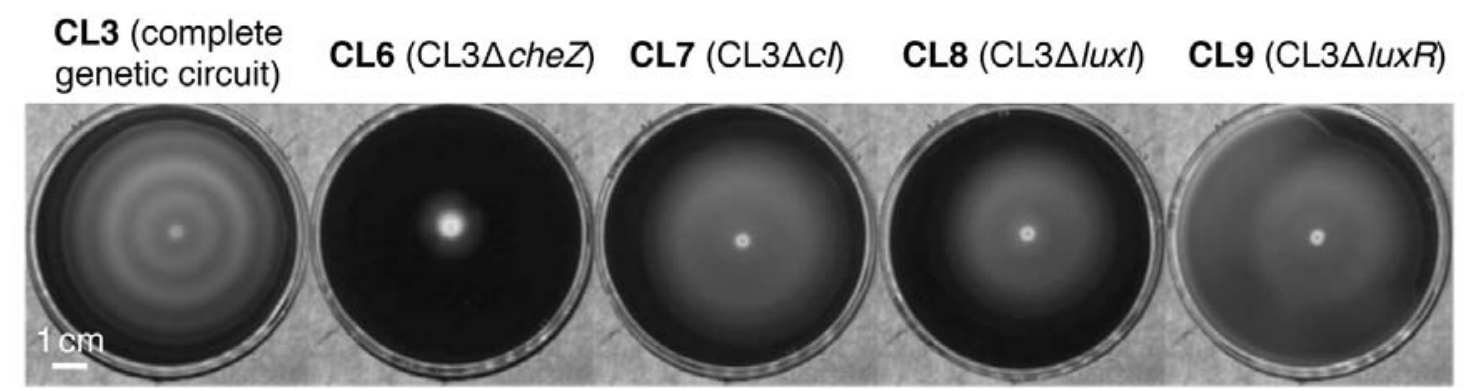

Fig. S1. The entire genetic circuit is required for stripe pattern formation. After seed culture and preculture, strains containing a complete (CL3) or partial genetic circuit (CL6, CL7, CL8, and CL9) were diluted 200-fold into fresh medium and cultured until the mid-exponential phase $\left(\mathrm{OD}_{600}=0.1-0.2\right)$ separately. $2-\mu 1$ cell suspension was then inoculated at the center of $0.23 \%$ agar plates, followed by 20 -h incubation at $37{ }^{\circ} \mathrm{C}$. Photographs were taken against a black background illuminated by an annular LED array from below. The property of each strain is indicated in the brackets. 
Figure S2

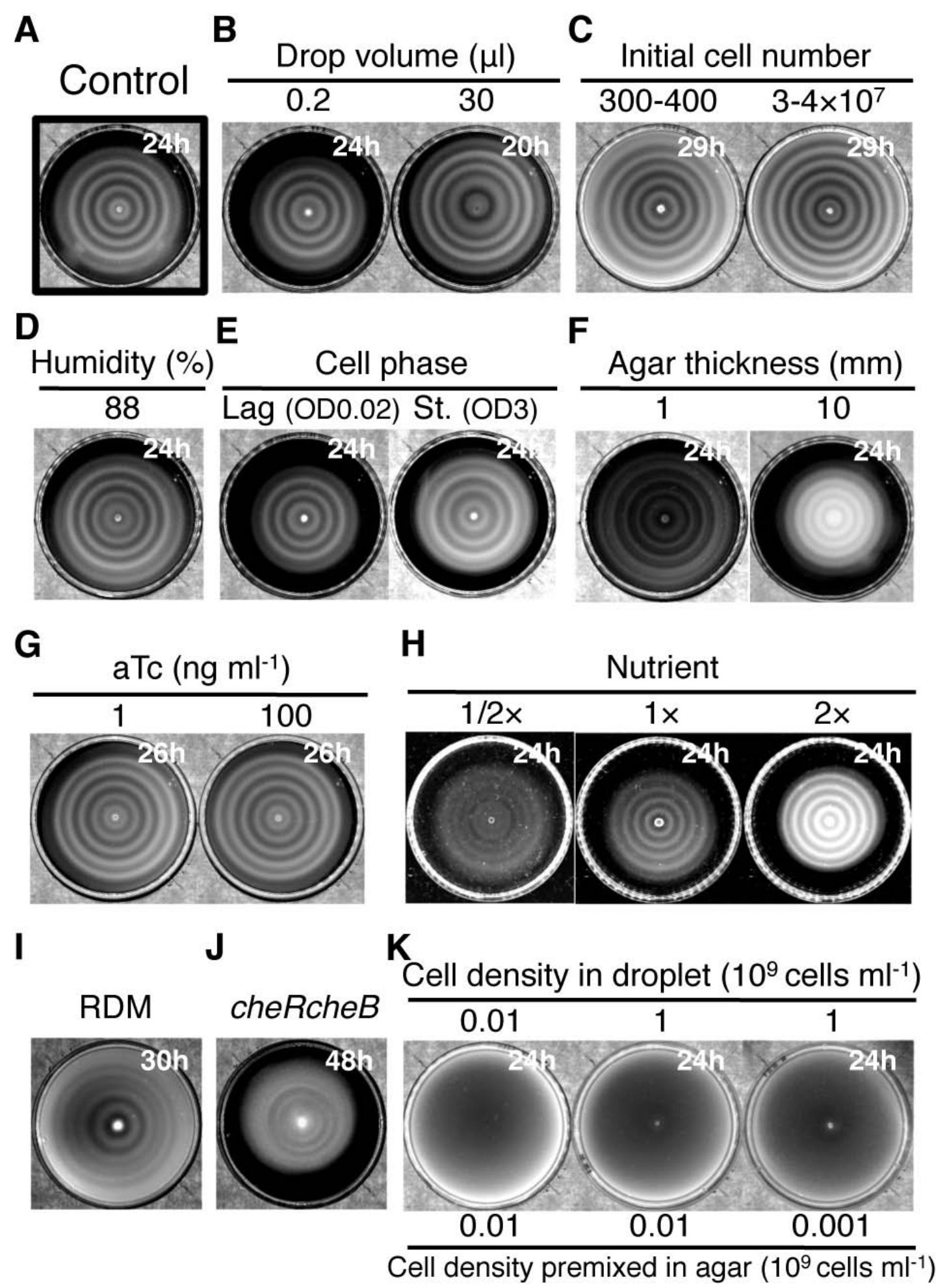

Figure S2. Pattern formation under various conditions. (A) The common experimental conditions applied in this study were used as the control, particularly, the initial drop volume was $2 \mu \mathrm{l}$; initial cell number was $3-4 \times 10^{5}$; initial cell phase was 
mid-exponential phase $\left(\mathrm{OD}_{600}=0.1-0.2\right)$; agar thickness was $2 \mathrm{~mm}$; and humidity was $30 \%$. (B) Initial drop volume from 0.2 to $30 \mu \mathrm{l}$ with the same cell density. Other conditions were kept unchanged. (C) Initial cell number, 1,000-fold dilution with experimental medium, or 100 -fold concentration by centrifugation. $2-\mu 1$ of cell suspension was inoculated. (D) Initial cell phase, after seed-culture, subculture, cells were 200-fold diluted into fresh experimental medium and grown until the lag-phase $\left(\mathrm{OD}_{600}=0.02\right)$, or stationary phase $\left(\mathrm{OD}_{600}=3\right)$. (E) Thickness of agar layer, LB medium supplemented with 0.1 M HEPES ( $\mathrm{pH}$ 8.0) containing $0.25 \%$ agar in various volumes, ranging from 5- to 50-ml, were poured into Petri dishes (8.5-cm internal diameter) and allowed to harden at room temperature for $90 \mathrm{~min}$. 2- $\mu$ l of cell suspension was inoculated. (F) Effect of humidity. The conditions were the same as the control except for $88 \%$ humidity. (G) Effect of aTc. After seed-culture, cells were subcultured into experimental medium supplemented with various concentrations of aTc, grown until the mid-exponential phase. $2-\mu 1$ of cell suspension was spotted at the center of semi-solid agar containing the medium with the same concentration of aTc as subculture. (H) Effect of the initial nutrient level. Keep $\mathrm{NaCl}$ and HEPES concentration unchanged, other ingredients of LB medium were diluted or concentrated two times. (I) Rich defined medium (RDM). The culture conditions were the same as the control except for the medium used (see Method S3). (J) cheR cheB double mutant. After seed-culture, subculture, CL15 cells (see Method S1 and Table S1) were inoculated onto LB medium supplemented with 0.1 M HEPES (pH 8.0) containing 0.13\% agar. (K) Initial cell densities in droplet and uniform mixture in agar. Cells were cultured until the mid-exponential phase, diluted or concentrated to the desired cell densities and mixed with pre-warmed $0.25 \%$ agar (as described in Method S3). Subsequently, cells in various densities were spotted in the center of the hardened cell-agar mixture. The times in the figures indicate the duration of the agar plate incubated at $37^{\circ} \mathrm{C}$ after cells were spotted. Pattern formation is robust in all the above conditions; however, conditions that significantly affect cell growth (e.g., temperature and nutrient) can affect the patterns in complex ways, presumably due to the cell's innate control on motility. Moreover, given larger space and longer time to develop patterns, "loss-of-pattern" mutations were observed. 
Figure S3

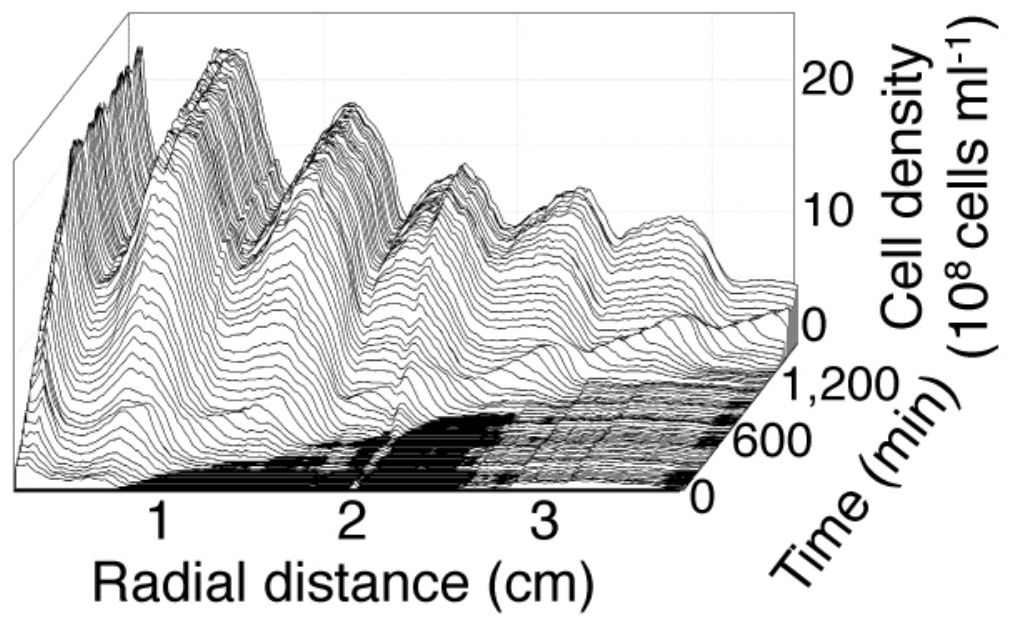

Fig. S3. Spatial-temporal cell density profile. To measure the absolute cell density profile across the center of the Petri dish as a function of time, the Petri dish was placed on a motorized stage, and the cell density profile was obtained by periodically scanning along a line through the center of the agar plate with a fine laser beam during the pattern formation process, and comparing the transmitted light with the standard; see Method S5 for details. 
Figure S4
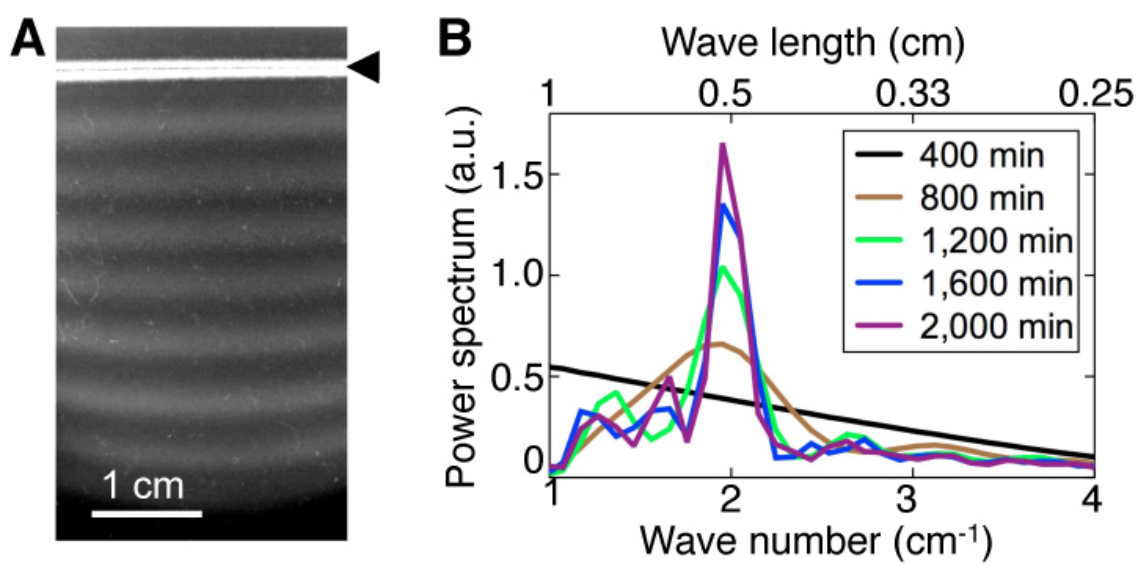

Fig. S4. One-dimensional pattern formed by strain CL3. (A) One-dimensional pattern observed 2,000 min after seeding. Arrow points to the position of inoculation (Method S7 and Movie S3). (B) Fourier analysis of one-dimensional band pattern formation. To evaluate the periodicity of the band pattern, the light intensity of the band pattern (A and Movie S3) was transformed from the real-space to the wave-number domain (see Method S9). The Fourier power spectra at various time points are shown. A peak around the wave number of $\sim 2 \mathrm{~cm}^{-1}$ appeared at time $800 \mathrm{~min}$, getting narrower and taller over time, indicating an expanding band pattern with a constant wavelength of $\sim 0.5 \mathrm{~cm}$. 
Figure S5

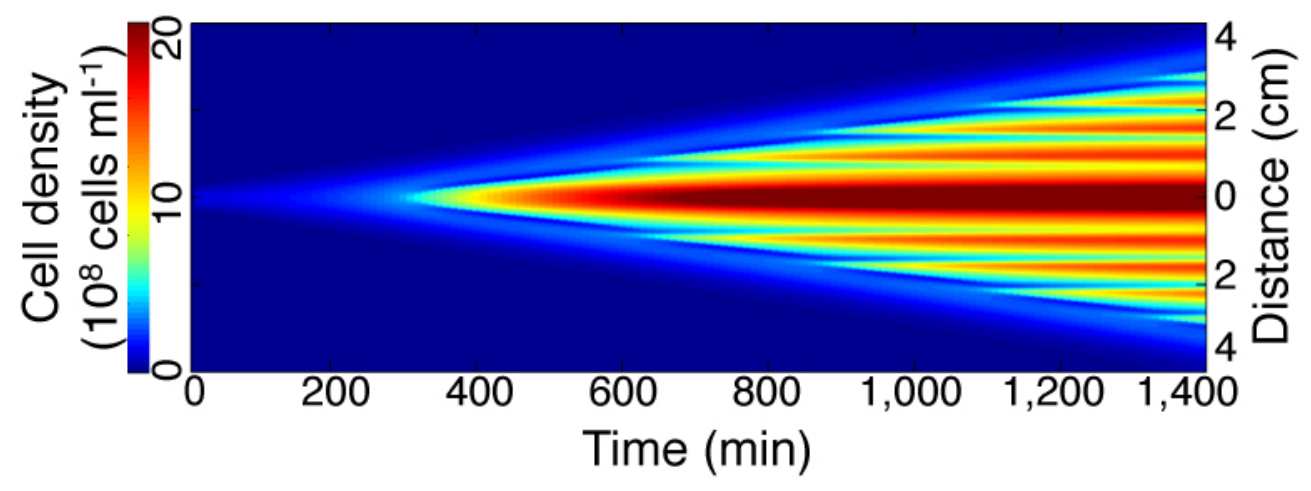

Fig. S5. Spatiotemporal diagram of cell density profile obtained from 1D simulation of Eqs. [1]-[3]. The simulation was done using the parameters in Table S3, starting with the initial condition as Eq. [S.15]. 
Figure S6
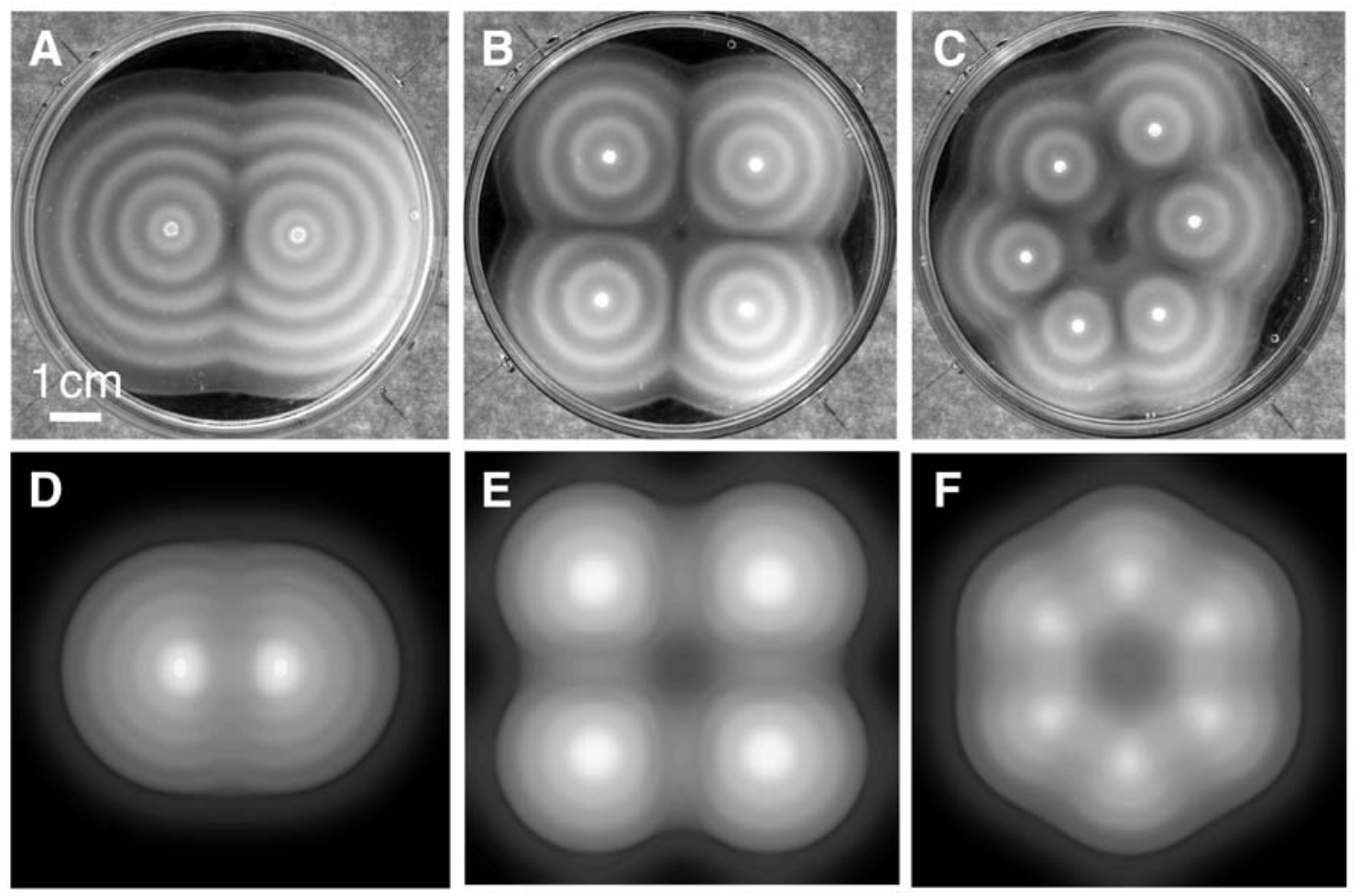

Fig. S6. Formation of intricate patterns. (A), (B), (C) Experimental results showing various patterns formed based on the placement of the inoculums. (D), (E), (F) Simulation results corresponding to (A), (B), and (C), respectively. 
Figure S7

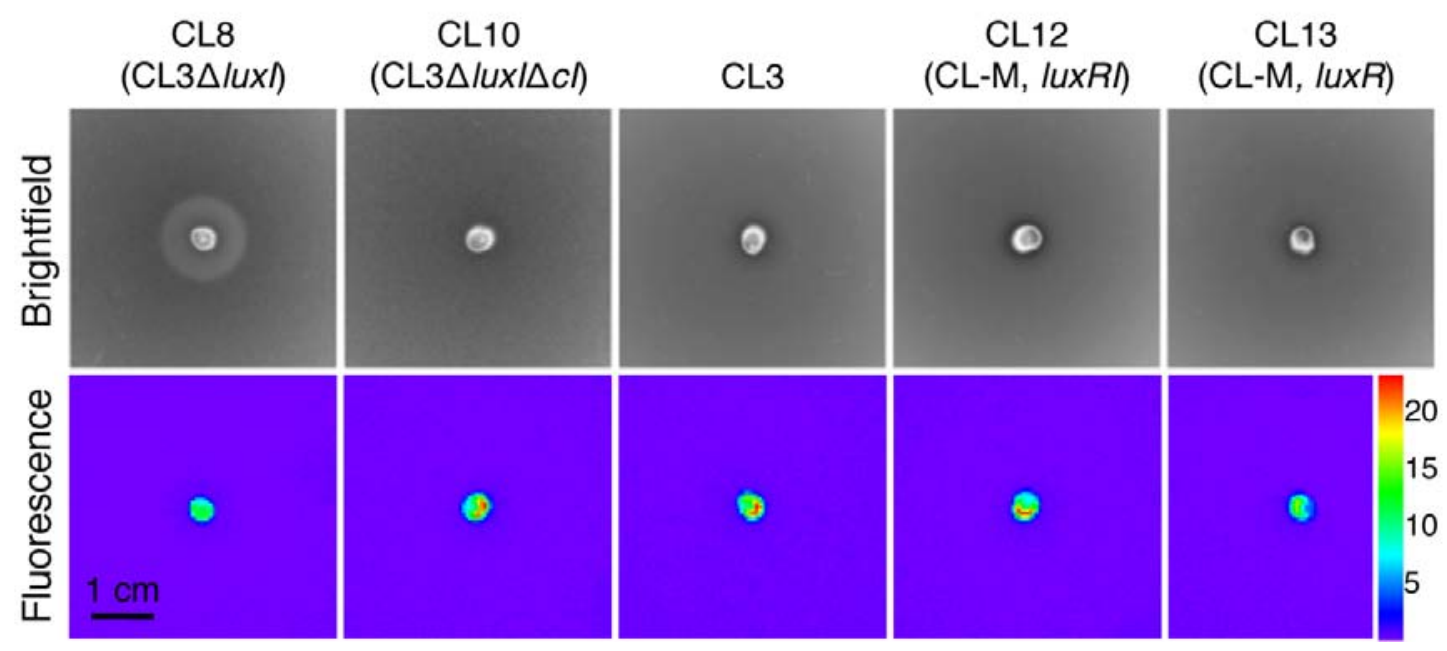

Fig. S7. Evidence of effective aggregation. To test the hypothesis of effective aggregation, various combinations of receiver and sender cells were conducted. All sender cells (CL6, non-motile but synthesizing AHL) were located at the position of inoculum as indicated by green fluorescence. Only those receiver cells with an entire genetic circuit but lacking the AHL-producing gene (luxI) formed a distinct cell aggregation around the spotted sender cells (CL8). Aggregation could not occur under other conditions, such as receiver cells lacking $c I$, which failed to regulate cheZ (CL10); receiver cells with AHL-producing ability, which might affect the formation of the AHL gradient (CL3); receiver cells with native cheZ and AHL-producing ability (CL12); receiver cells with native cheZ but incapable of AHL synthesis (CL13). Upper panels represent bright field images. Lower panels represent corresponding fluorescence images. 
Figure S8

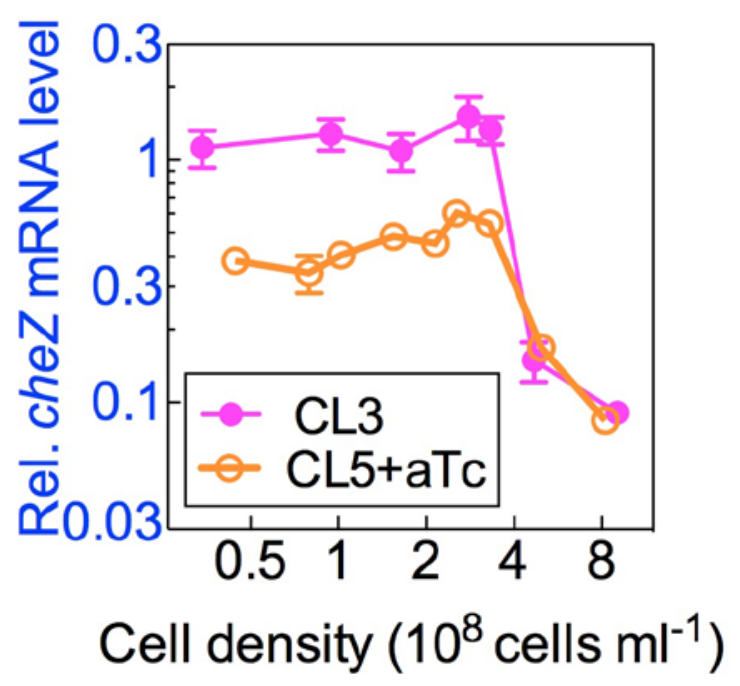

Fig. S8. Relative cheZ mRNA levels of CL5 as a function of cell density in bulk culture supplemented with $0.4 \mathrm{ng} \mathrm{ml}^{-1}$ aTc. CL3 was included as a control. Data are normalized by the mean value of CL3 at $0.3 \times 10^{8}$ cells $\mathrm{ml}^{-1}$. 
Figure S9
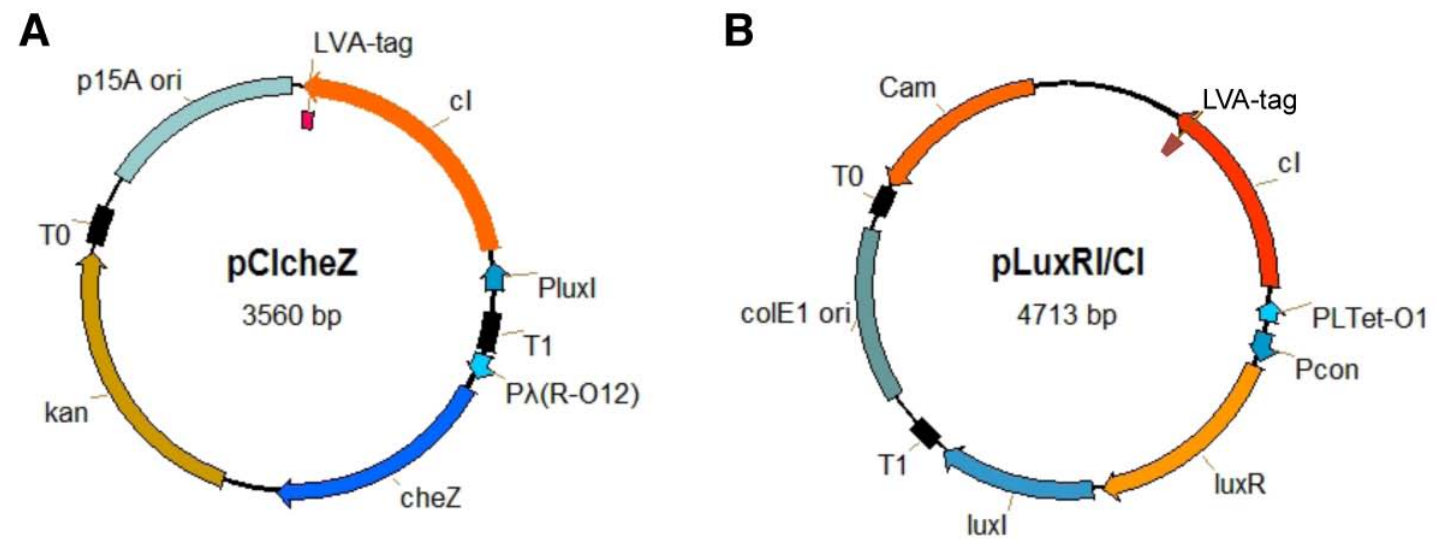

Fig. S9. Plasmids for the tunable stripe pattern formation. (A) pCIcheZ. cheZ and $c I$ are under the control of CI repressible promoter $P_{\lambda(\mathrm{R}-\mathrm{O} 12)}$ and cell density-inducible promoter $P_{\text {luxi }}$, respectively. They are oriented back to back. (B) pLuxRI/CI. luxR and luxI are constitutively expressed (see Method S2 for details). The $c I$ gene is driven by a

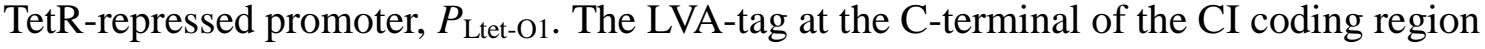
will facilitate the turnover of $\mathrm{CI}$ in the cells (62). T0 and $\mathrm{T} 1$ indicate transcriptional terminators. 
Figure S10

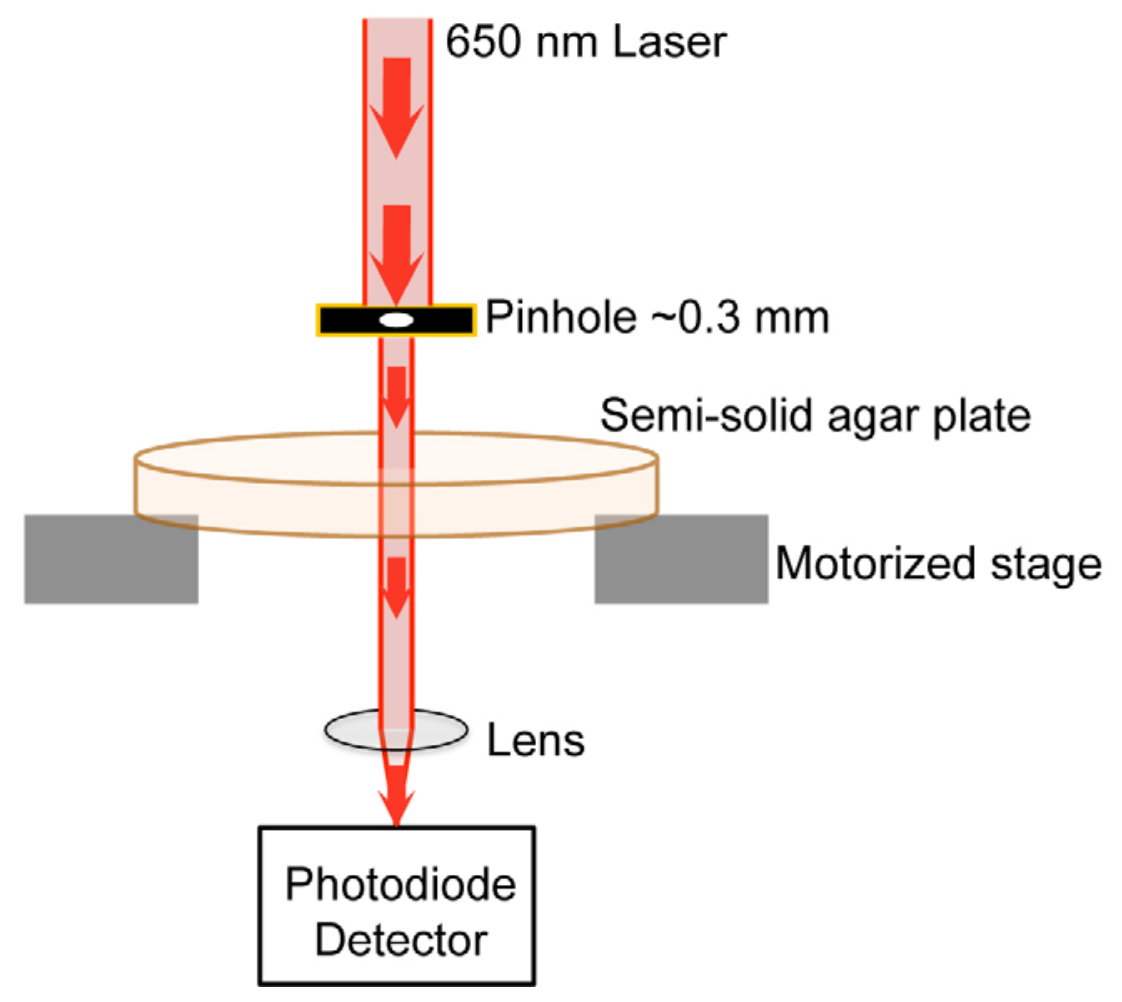

Fig. S10. The setup of the customized device for the real-time measurement of the spatiotemporal cell density profile in semi-solid agar dishes. A parallel 650-nm laser beam is guided through a 300- $\mu \mathrm{m}$ pinhole, passed through the sample in semi-solid agar on the Petri dish, and collected via a convex lens to a photodiode detector. The light intensity is digitized with a DAQ device and stored on a personal computer. The spatiotemporal scanning is realized with a motorized stage controlled with the PC. The whole apparatus was placed in a warm room $\left(37^{\circ} \mathrm{C}\right)$ throughout the experiment. 
Figure S11
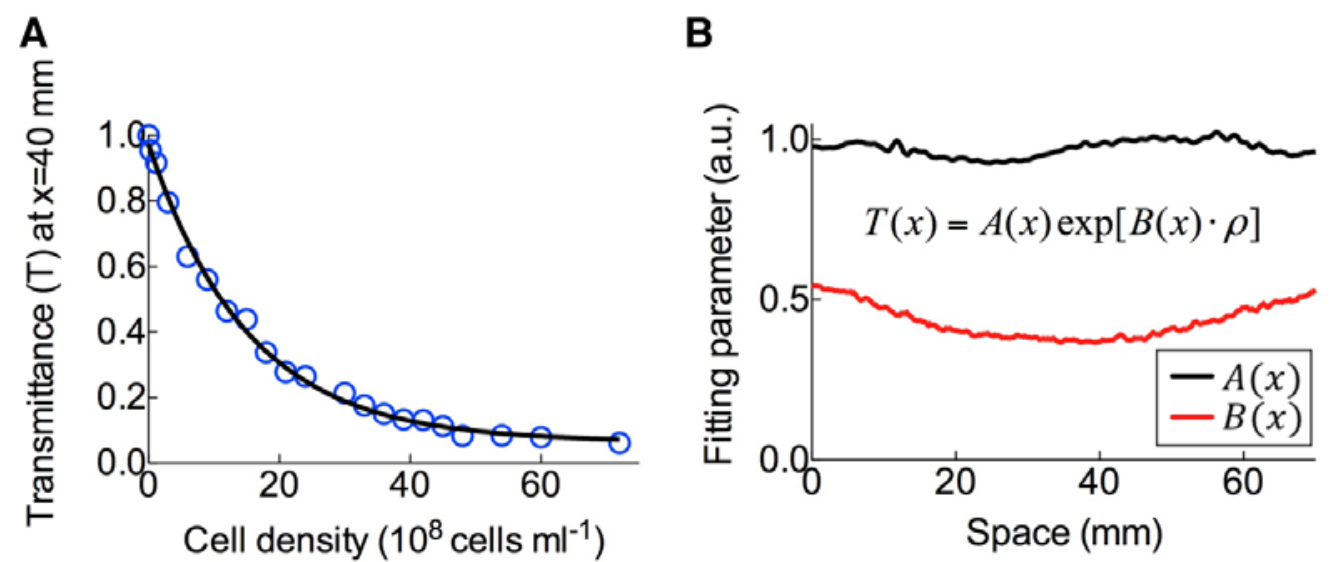

Fig. S11. The calibration curve of light transmittance vs. real cell density in semi-solid agar plates. (A) An example of the fitted curve of the transmittance as the function of cell density at a specific position. (B) The spatial distribution across the center of the dish, of the two fitting parameters for the standard curve, $A(x)$ (black line) and $B(x)$ (red line). This is derived from 376 standard curves (scanning range $=75 \mathrm{~mm}$; step size $=$ $0.2 \mathrm{~mm})$. 
Figure S12

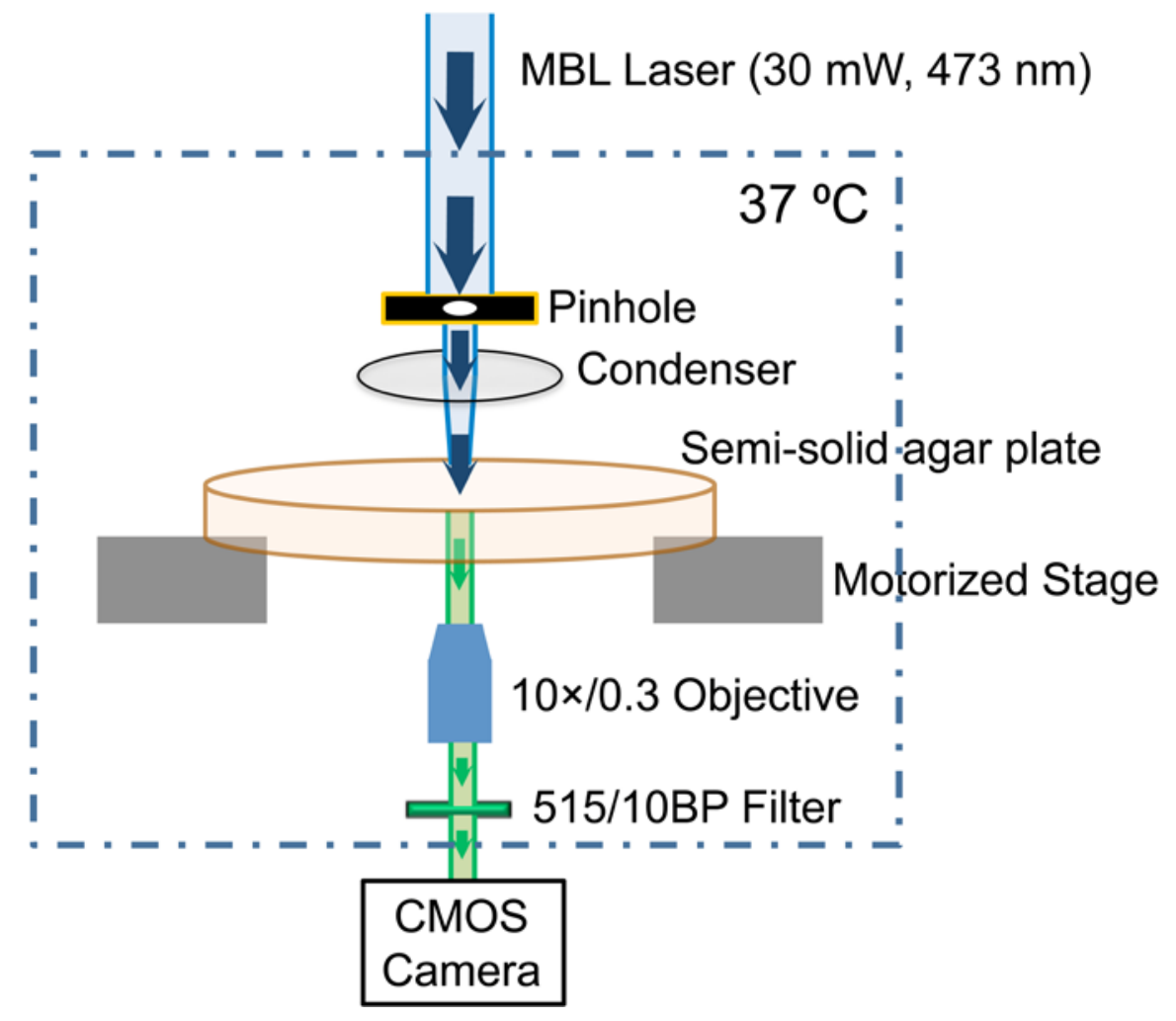

Fig. S12. A schematic diagram for the continuous fluorescence photobleaching (CPB) microscopy system. A parallel 473-nm laser beam is guided through a pinhole and a condenser to excite the GFP molecules in the cells in a cylindrical volume. The emitted fluorescence is collected via an objective lens, passed through a narrow band green filter, and detected with a CMOS camera. Throughout the measurement, the semi-solid agar plate was placed on a motorized stage within an environmental chamber, which is kept constant at $37^{\circ} \mathrm{C}$. 
Figure S13
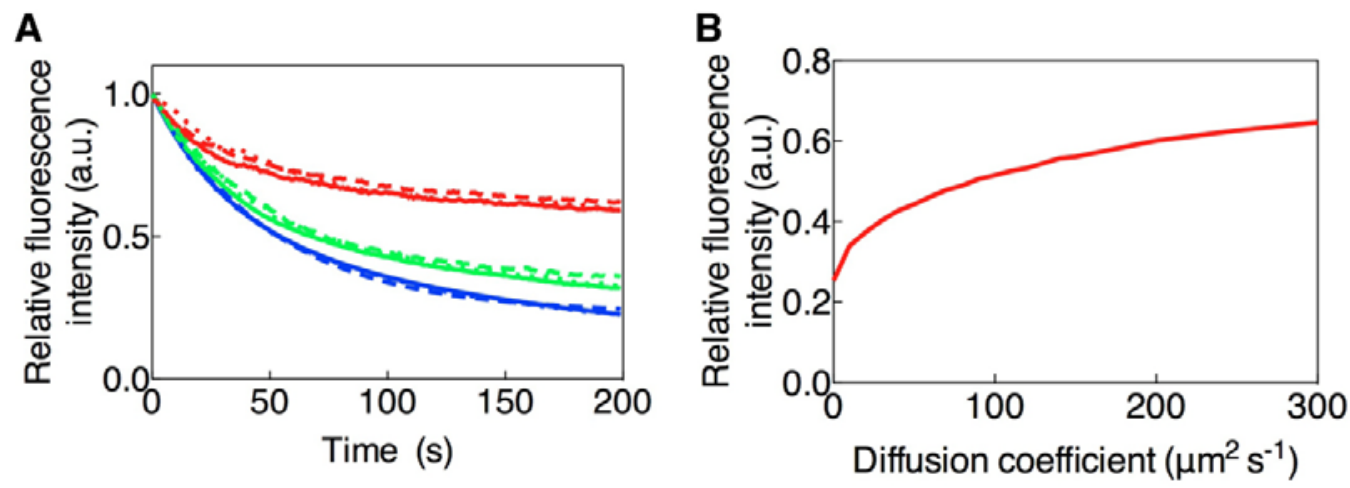

Fig. S13. Measurement of cell diffusion coefficient via continuous fluorescence photobleaching (CPB). (A) The representative CPB curves of different diffusion coefficients. Blue dots are experimental data of immobile strain CL14. Red and green dots are representative data of CL3GFP with fast and slow diffusion coefficients, respectively. Blue lines are fitting curves of blue dots $\left(D=0 \mu \mathrm{m}^{2} \mathrm{~s}^{-1}\right)$ using double component decay function $f(t)=0.48 e^{0.03 t}+0.52 e^{0.004 t}$ (solid) and single decay function $f^{\prime}(t)=0.75 e^{0.018 t}+0.25$ (dashed). Red and green lines are the simulation results of $D=110 \mu \mathrm{m}^{2} \mathrm{~s}^{-1}$ and $D=5 \mu \mathrm{m}^{2} \mathrm{~s}^{-1}$, respectively, with the corresponding blue lines as $D=0$ standard. (B) The relationship between diffusion coefficient and relative fluorescence intensity (mean value from 175 to $180 \mathrm{~s}$ ) by using $f(t)$ as the $D=0$ standard. 
Figure S14

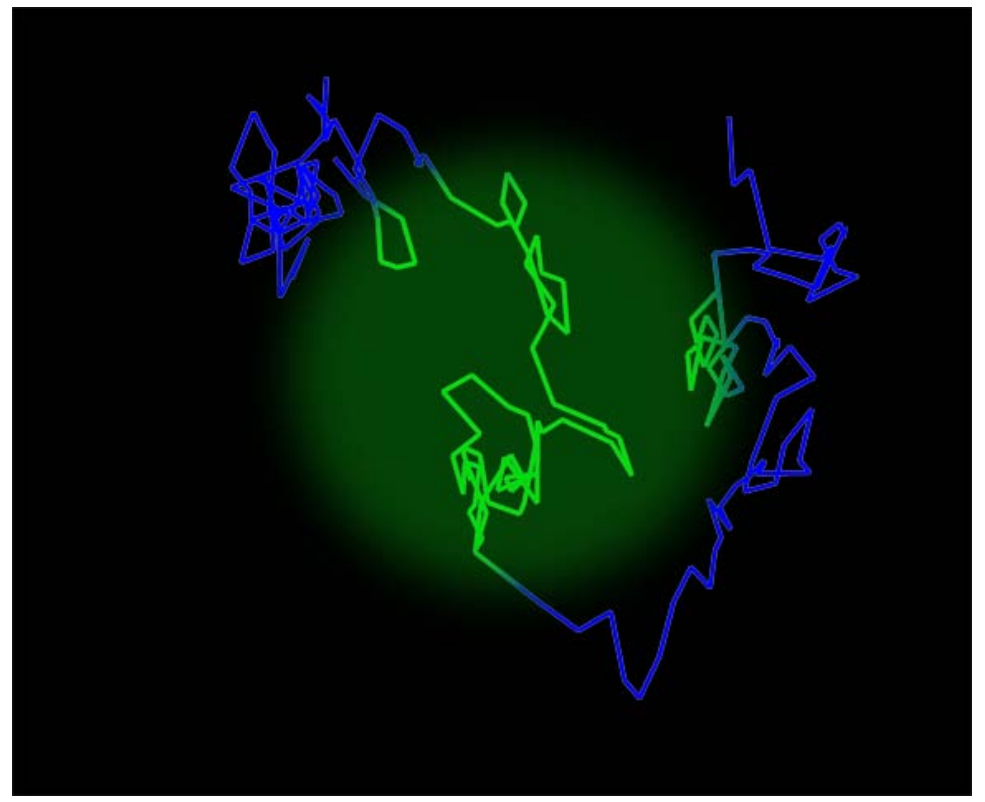

Fig. S14. Illustration of the trajectory of one cell in and out of the photobleaching region. The line represents a simulated 2D random walk trajectory of an E. coli cell. The GFP molecules would undergo photobleaching only when the cell locates in the laser-illuminated region (green). The accumulated photobleaching time $(\tau)$ was derived from the portions of the trajectory in the green region. 
Figure S15

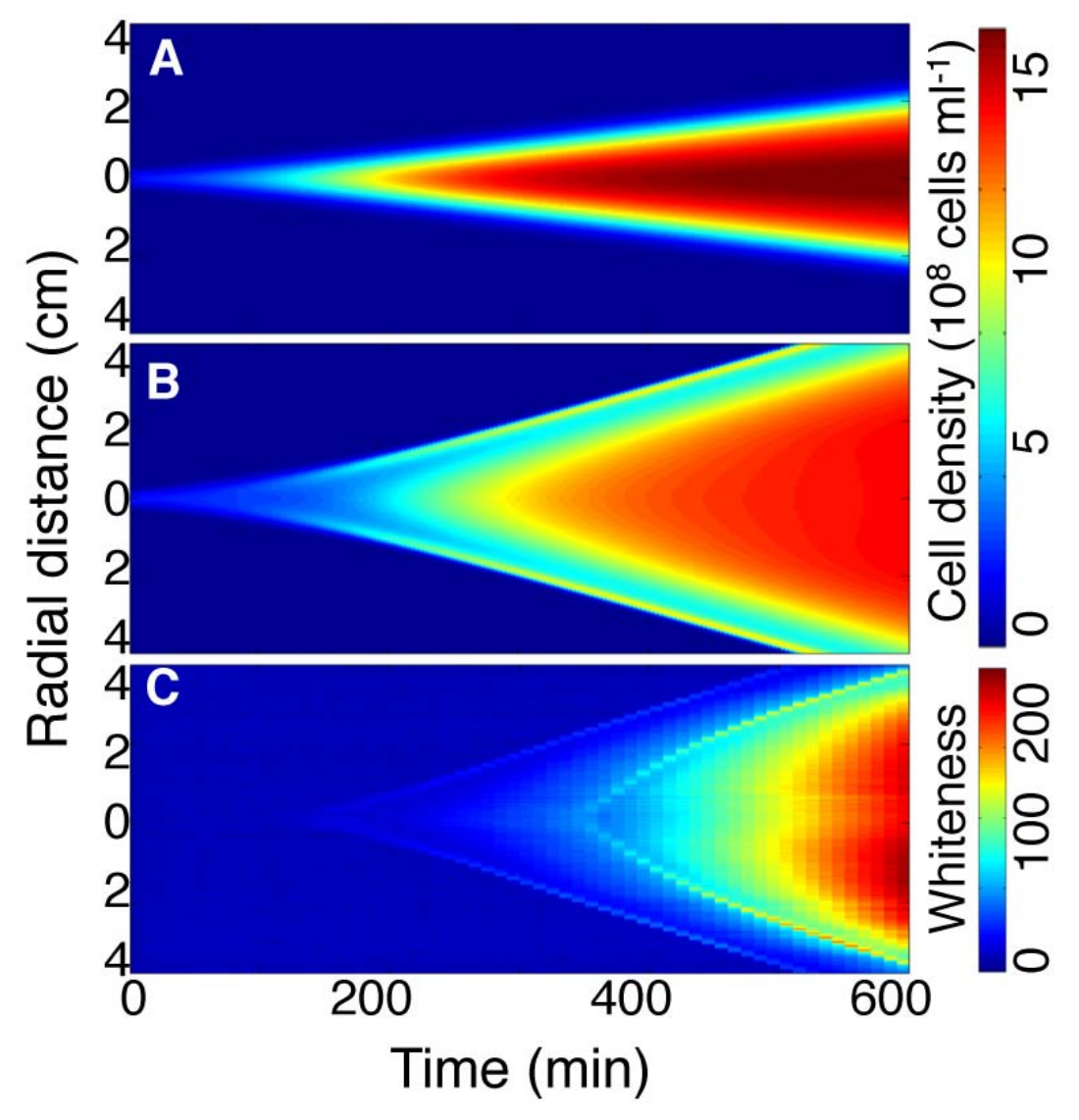

Fig. S15. The simulated spatiotemporal density profile in 1D. (A) without chemotaxis (Eqs.[S.5] -[S.7] with $D_{\rho}=450 \mu \mathrm{m}^{2} \mathrm{~s}^{-1}, \gamma \sim 1.4 \mathrm{~h}^{-1}, K_{C_{1}}=0, K_{n}=1 \times 10^{9}$ cells $\mathrm{ml}^{-1}$, $k_{n}=1$, and $n(t=0)=1.5 \times 10^{9}$ cells $\left.\mathrm{ml}^{-1}\right)$. (B) with chemotaxis $\left(K_{C_{1}}=2 \times 10^{9}\right.$ cells $\mathrm{ml}^{-1}, K_{C_{2}}=1.5 \times 10^{8}$ cells $\mathrm{ml}^{-1}, K_{a}=0.1 \times 10^{9}$ cells $\mathrm{ml}^{-1}, k_{a}=1$ and $a(t=0)=0.35 \times 10^{9}$ cells $\mathrm{ml}^{-1}$ ). The drift term changes the Fisher's wave front shape and increases its propagation speed. (C) The experimental spatiotemporal diagram of CL4 (harboring quorum-sensing module but with native cheZ regulation), showing the light intensity along a vertical line through the center of the Petri dish as a function of time. 
Figure S16

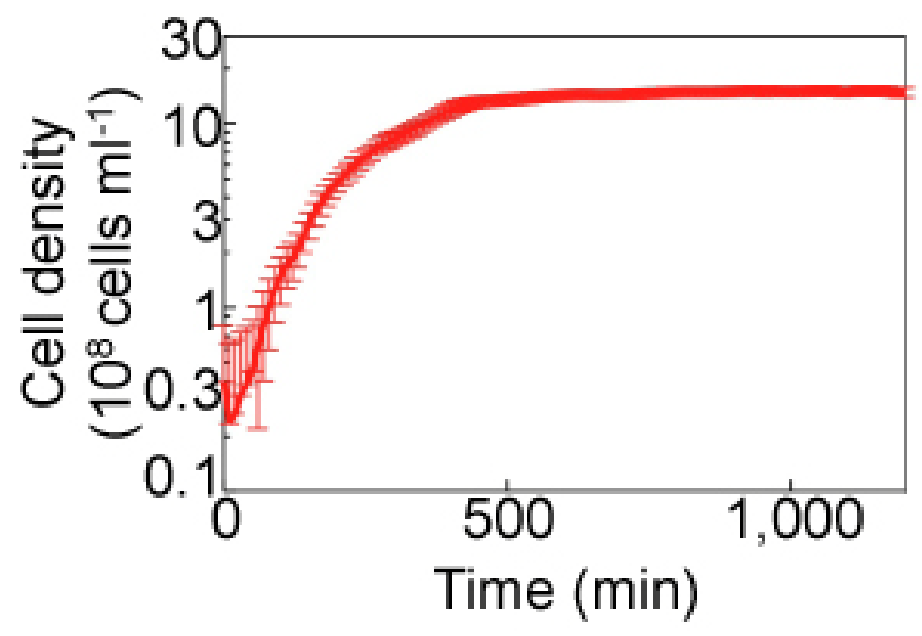

Fig. S16. Growth curve of CL3 in a Petri dish containing 10-ml 0.25\% LB agar. After seed culture and preculture growth, CL3 cells were diluted 200-fold into the pre-warmed LB media containing $0.25 \%$ agar. Cell-agar mixtures were poured into Petri dishes and allowed to harden at room temperature for $90 \mathrm{~min}$, and then moved back into a $37^{\circ} \mathrm{C}$ incubator. The cell densities in the semi-solid agar dish were automatically measured every 10 min (see Method S5). 
Figure S17

A

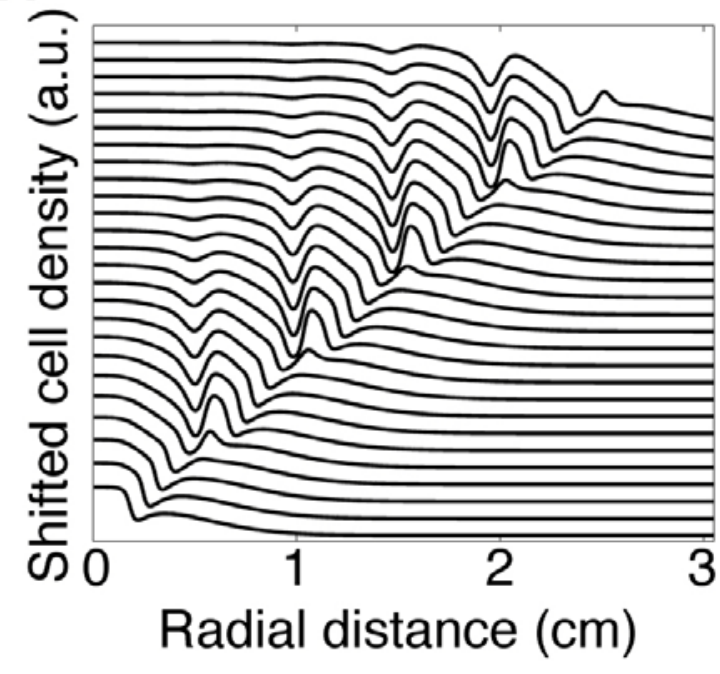

B

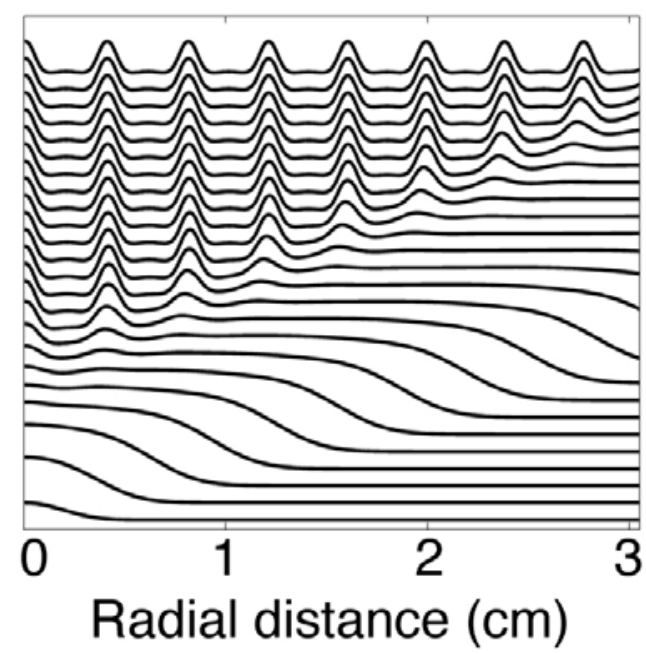

Fig. S17. The simulated cell density profile of Eqs. [S.11] and [S.12]. The y-axis represents the cell density, (shifted every $30 \mathrm{~min}$ ). (A) simulated pattern with $D_{\rho}=500$ $\mu \mathrm{m}^{2} \mathrm{~s}^{-1}, \gamma \sim 0.7 \mathrm{~h}^{-1}, \rho_{\mathrm{s}}=1.5 \times 10^{9}$ cells $\mathrm{ml}^{-1}, \alpha=\beta: 2 \mathrm{~h}^{-1}$, and $K_{h}=4 \times 10^{8}$ cells $\mathrm{ml}^{-1}$. (B) simulated pattern with $D_{\rho}=100 \mu \mathrm{m}^{2} \mathrm{~s}^{-1}$, and $K_{h}=1.5 \times 10^{9}$ cells ml $^{-1}$. 
Figure S18

Cell density premixed in agar (cells $\mathrm{ml}^{-1}$ )

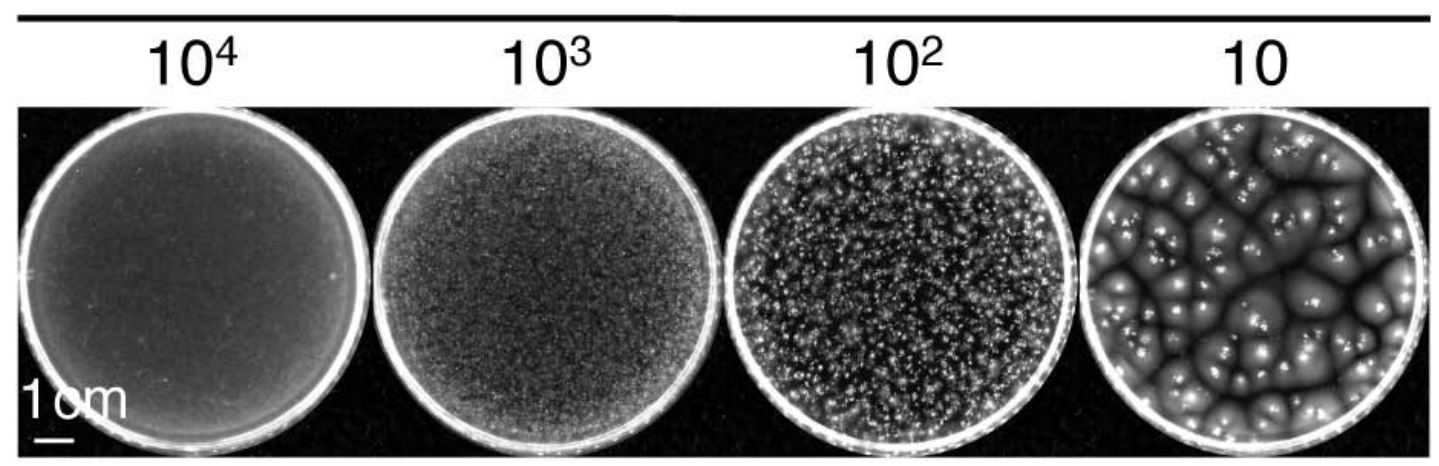

Fig. S18. Patterns formed by mixing agar with cells in various initial cell densities. Cells were cultured until the mid-exponential phase, diluted or concentrated to desired cell densities and mixed with pre-warmed 0.25\% agar (see Method S3 for details), followed by 20 -h incubation at $37^{\circ} \mathrm{C}$. Random patches of cells were formed with no periodic patterns. Scale bar, $1 \mathrm{~cm}$. 
Figure S19

A

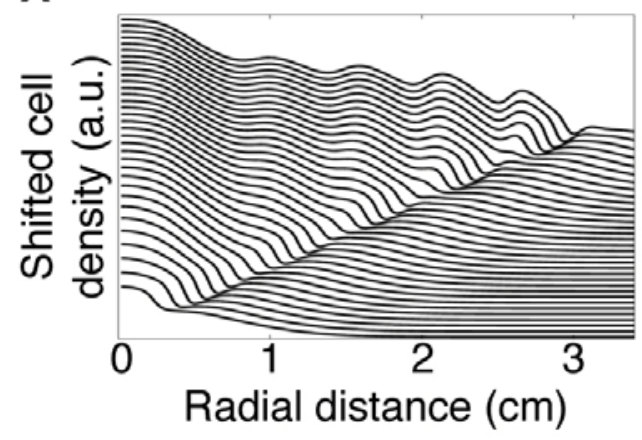

B

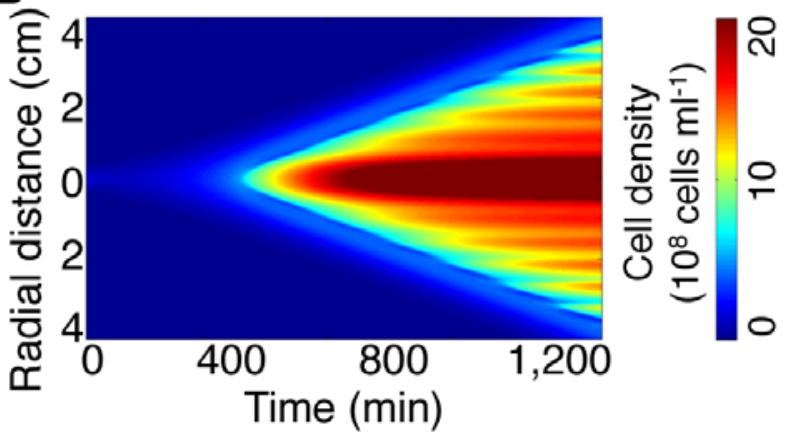

Fig. S19. The 2D simulation of Eqs.[S.12]-[S.14] with $D_{\rho}=500 \mu \mathrm{m}^{2} \mathrm{~s}^{-1}$. (A) The y-axis represent the cell density profile (shifted every $30 \mathrm{~min}$ ). (B) The simulated spatiotemporal density profile. 
Figure S20
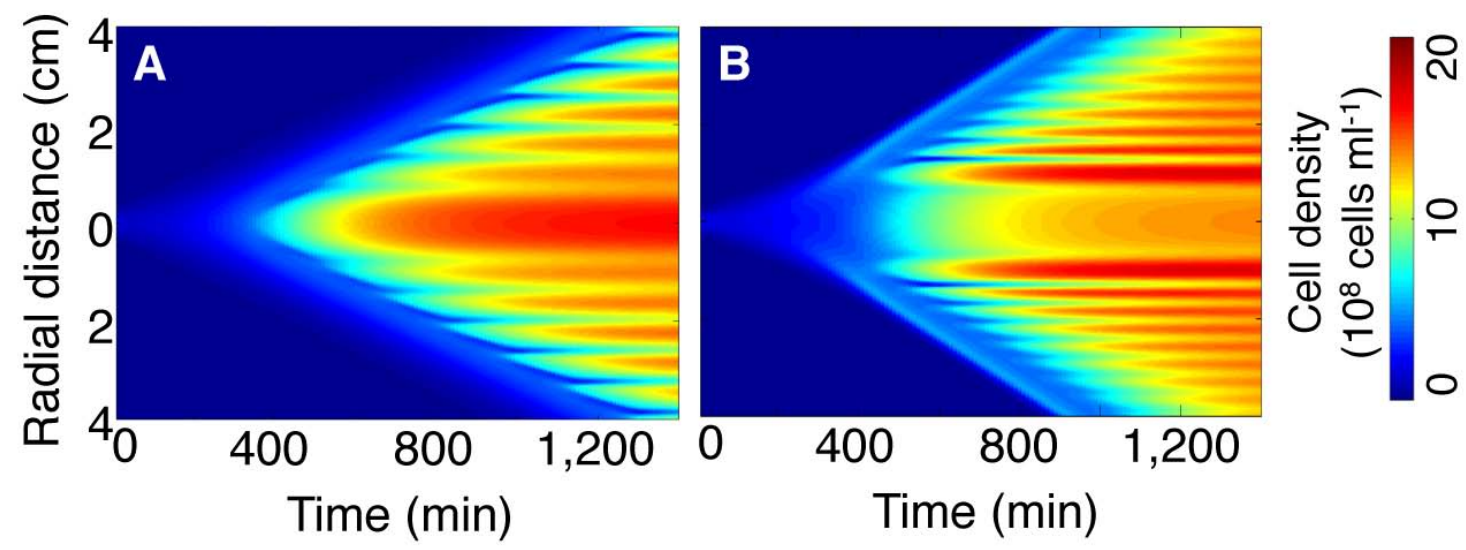

Fig. S20. The simulated spatiotemporal density profiles. (A) Eqs.[S.12], [S.14] and [S.16] with $D_{\rho}=500 \mu \mathrm{m}^{2} \mathrm{~s}^{-1}$ and $\alpha=\beta: 2 \mathrm{~h}^{-1}$. (B) Eqs. [S.6], [S.12] and [S.14] and [S.17] with $D_{\rho}=450 \mu \mathrm{m}^{2} \mathrm{~s}^{-1} \alpha=\beta: 1.4 \mathrm{~h}^{-1}, K_{C_{1}}=2 \times 10^{9}$ cells $\mathrm{ml}^{-1}, K_{C_{2}}=1.5 \times 10^{8}$ cells $\mathrm{ml}^{-1}, K_{a}=0.1 \times 10^{9}$ cells $\mathrm{ml}^{-1}, k_{a}=1$ and $a(\mathrm{t}=0)=0.35 \times 10^{9}$ cells ml $^{-1}$. 
Figure S21

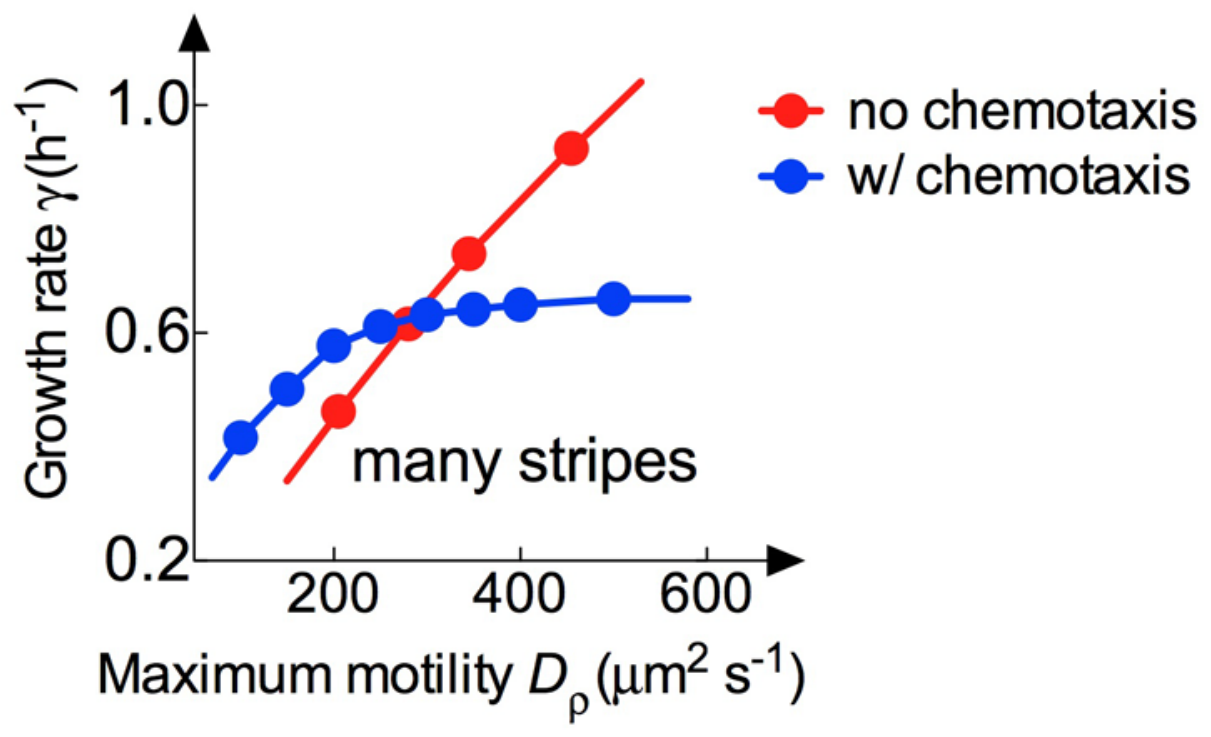

Fig. S21. The phase boundary of the stripe pattern formation. The behavior of this system is controlled mostly by the two dimensionless combinations of parameters, $D_{\rho} / D_{h}$ and $\gamma / \beta$. Since the AHL diffusion coefficient and half-life is not easy to be modified experimentally, we fixed the $D_{h}\left(400 \mu \mathrm{m}^{2} \mathrm{~s}^{-1}\right)$ and $\beta\left(1.4 \mathrm{~h}^{-1}\right)$ and simulate the phase boundary by varying the maximum cell motility $D_{\rho}$ and the growth rate $\gamma$ with the initial condition described with Eq.[S.18]. The red dots represent the phase boundary without the chemotaxis term (same as in Fig. 4A). The system can only form many stripes below this boundary. With the model including chemotaxis (Eqs. [S.6], [S.12], [S.14] and [S.17] with $K_{C_{1}}=2 \times 10^{9}$ cells $\mathrm{ml}^{-1}, K_{C_{2}}=1.5 \times 10^{8}$ cells ml ${ }^{-1}$, $K_{a}=0.1 \times 10^{9}$ cells $\mathrm{ml}^{-1}, k_{a}=1$ and $a(\mathrm{t}=0)=0.35 \times 10^{9}$ cells $\mathrm{ml}^{-1}$ ), the phase boundary shifted a little. The detailed position of this boundary can also be affected by the other parameters, such as $K_{C_{1}}, K_{C_{2}}, K_{a}$, etc. 


\section{SUPPLEMENTARY TABLES}

Table S1 Strains and plasmids used in this study

\begin{tabular}{|c|c|c|}
\hline Strains or plasmids & Genotype or description & Reference or source \\
\hline \multicolumn{3}{|l|}{ Strains } \\
\hline CL-M & A motile wild type E. coli K12 strain AMB 1655 & $\begin{array}{l}\text { Prof. Danchin } \\
\text { (AMAbiotics, France) }\end{array}$ \\
\hline CL-IM & A immotile E. coli K12 strain MG1655 & $\begin{array}{l}\text { Prof. Danchin } \\
\text { (AMAbiotics, France) }\end{array}$ \\
\hline CL1 & $\Delta c h e Z, \Delta l a c$ & CL-M, this study \\
\hline CL1RB & $\Delta$ cheZ, $\Delta l a c, \Delta c h e R, \Delta c h e B$ & CL1, this study \\
\hline CL2 & $\Delta c h e Z, \Delta l a c, b l a: P_{\text {Ltet-O1-tet } R \text { at the } a t t B \text { site }}$ & CL1, this study \\
\hline CL3 & pCIcheZ, pLuxRI2 & CL1, this study \\
\hline CL3GFP & pCIcheZ, pLuxRI/GFP & CL1, this study \\
\hline CL4 & pLuxRI/GFP & CL-M, this study \\
\hline CL5 & pCIcheZ, pLuxRI/CI & CL2, this study \\
\hline CL6 & pCIcheZ $\Delta$ cheZ, pLuxRI/GFP & CL1, this study \\
\hline CL7 & pCIcheZ $\Delta$ cI, pLuxRI2 & CL1, this study \\
\hline CL8 & pCIcheZ, pLuxR & CL1, this study \\
\hline CL9 & pCIcheZ, pLuxI & CL1, this study \\
\hline CL10 & pCIcheZ $\Delta$ cI, pLuxR & CL1, this study \\
\hline CL11 & pCIcheZ $\Delta$ cheZ, pLuxR/GFP & CL1, this study \\
\hline CL12 & pCIcheZ $\Delta$ cheZ, pLuxRI2 & CL-M, this study \\
\hline CL13 & pCIcheZ $\Delta$ cheZ, pLuxR & CL-M, this study \\
\hline CL14 & pLuxRI/GFP & CL-IM, this study \\
\hline CL15 & pCIcheZ, pLuxRI2 & CL1RB, this study \\
\hline EQ44 & $\Delta l a c Y$, bla: $\mathrm{P}_{\text {LTet-O1 }}$-tet $R$ at the $a t t B$ site & (35) \\
\hline
\end{tabular}

\section{Plasmids}

\begin{tabular}{|c|c|c|}
\hline pSim6 & $\mathrm{Ap}^{\mathrm{r}}, \mathrm{pSC101}$ ori, $\lambda$ Red & $(34)$ \\
\hline pEGFP-loxP-CmR-loxP & $\mathrm{Ap}^{\mathrm{r}}, \mathrm{Cm}^{\mathrm{r}}$, pUC ori, loxp-Cm ${ }^{r}-$ loxp & (33) \\
\hline p705cre & $\mathrm{Km}^{\mathrm{r}}$, pSC101 ori, cre & $(63)$ \\
\hline pLuxRI2 & $\mathrm{Cm}^{\mathrm{r}}$, ColE1 ori, Pcon-luxR-luxI & $(36)$ \\
\hline pLD & $\mathrm{Km}^{\mathrm{r}}, \mathrm{p} 15 \mathrm{~A}$ ori, $P_{\lambda(0-12)^{-}}$lacI, $P_{\mathrm{lux} I}-c I$ & $(9)$ \\
\hline pCIcheZ & $\mathrm{Km}^{\mathrm{r}}$, p15A ori, $P_{\lambda(0-12)^{-}}$cheZ, $P_{\text {luxi }}-c I$ & This study \\
\hline pLuxRI/CI & pLuxRI2 carring $P_{\text {tet }}-\mathrm{CI}$ & This study \\
\hline
\end{tabular}




\begin{tabular}{lll} 
pLuxRI/GFP & pLuxRI2 carring Pcon-superfolder gfp & This study \\
pLuxR/GFP & pLuxR carring Pcon-superfolder gfp & This study \\
pCIcheZ $\Delta$ cheZ & pCIcheZ lacking cheZ-coding region & This study \\
pCIcheZ $\Delta$ cI & pCIcheZ lacking $c I$-coding region & This study \\
pLuxR & pLuxRI2 lacking luxI-coding region & This study \\
pLuxI & pLuxRI2 lacking luxR-coding region & This study \\
pCIcheZ16S & pCIcheZ carring a copy of $16 \mathrm{~S}$ rRNA gene & This study \\
\hline
\end{tabular}

Table S2 Oligonucleotides used in this study

\begin{tabular}{|c|c|c|}
\hline Name & Sequence & Use \\
\hline cheZ-del-f & $\begin{array}{l}\text { ggaaaaactcaacaaaatctttgagaaactgggcatgtga } \\
\text { ggatgcgactctcgagccgatcatattcaataa }\end{array}$ & cheZ deletion \\
\hline cheZ-del-r & $\begin{array}{l}\text { ttatcagaccgcctgatatgacgtggtcacgccacatcag } \\
\text { gcaatacaaagacctcgaggactagtgaacctc }\end{array}$ & cheZ deletion \\
\hline cheZ-del-conf-f & aactgggcatgtgaggat & Confirmation \\
\hline cheZ-del-conf-r & gccacatcaggcaataca & Confirmation \\
\hline lacIZYA-del-f & $\begin{array}{l}\text { gcggtatggcatgatagcgcccggaagagagtcaattca } \\
\text { gggtggtgaatctcgagccgatcatattcaataac }\end{array}$ & lac operon deletion \\
\hline lacIZYA-del-r & $\begin{array}{l}\text { ttcatgccggatgcggctaatgtagatcgctgaacttgtag } \\
\text { gcctgataagacctcgaggactagtgaacctc }\end{array}$ & lac operon deletion \\
\hline lac-del-conf-f & ggcagaatgcttaatgaatta & Confirmation \\
\hline lac-del-conf-r & ttcctgcgctttgttcat & Confirmation \\
\hline cheRB-bla-f & $\begin{array}{l}\text { tgcagttacaaattgcgccagtggtatcctgaagtgattga } \\
\text { gaaggcgctatgagtattcaacatttccgtgt }\end{array}$ & cheRcheB deletion \\
\hline cheRB-bla-r & $\begin{array}{l}\text { atccacaaccaaaaatttaagttctttatccgccatttcaca } \\
\text { ctcctgatttaccaatgcttaatcagtgagg }\end{array}$ & cheRcheB deletion \\
\hline bla-conf-f & aatagtgtatgcggcgacc & Confirmation \\
\hline cheRB-conf-r & tgtggcggtatttaccett & Confirmation \\
\hline cheZ-f & atgatgcaaccatcaatcaaa & Cloning cheZ into pLD \\
\hline cheZ-r-Nhe & ctagctagctcaaaatccaagactatccaac & Cloning cheZ into pLD \\
\hline pLD-rot-f-xba & gctctagagtttttccataggctccgc & Linearing pLDcheZ flanking $P_{\text {lux }}-C I$ \\
\hline pLD-rot-r-cla & ccatcgatgagtaggacaaatccgccg & Linearing pLDcheZ flanking $P_{\text {luxi }}-C I$ \\
\hline cI-f-cla & ccatcgatgctttacgttcacactattgtatcg & Cloning $P_{\text {lux }}-C I$ into linearrized pLDcheZ \\
\hline cI-r-xba & gctctagaagaacaccttgccgatcattaa & Cloning $P_{\text {lux }}-C I$ into linearrized pLDcheZ \\
\hline cI-f-sca & aaaagtactgagaatttcacacaggggtac & Cloning $c I$ into pCRT-1 \\
\hline cI-r-not & aaagcggccgctagaagaacaccttgccga & Cloning $c I$ into pCRT- 1 \\
\hline gfp-f-sac & $\begin{array}{l}\text { cgagctcttgacggctagctcagtcctaggtacagtgcta } \\
\text { gcttgggctagctactagagaaagag }\end{array}$ & $\begin{array}{l}\text { Cloning superfolder-gfp into pLuxRI2, } \\
\text { the synthetic promoter is underlined }\end{array}$ \\
\hline gfp-r-sac & cgagctcttatcatcatttgtacagttcatcc & Cloning superfolder-gfp into pLuxRI2 \\
\hline gfp-conf-r & acgcacggaaaacttatgac & confirmation \\
\hline delcI-f-cla & acaatagtgtgaacgtaaagcatc & Deleting $c I$ from pCIcheZ \\
\hline delcI-r-cla & gcatcgatcggcaaggtgttcttctag & Deleting $c I$ from pCIcheZ \\
\hline
\end{tabular}




\begin{tabular}{ll} 
16S-f-cla & ccatcgatattgaagagttgatcatggc \\
16S-r-xho & ccgctcgagaggaggtgatccaaccg \\
RT-cheZ-f & ctggaaatcatgatggcg \\
RT-cheZ-r & tgctggtatcgacctgagg \\
RT-cI-f & ggttagtatgcagccgtcactt \\
RT-cI-r & caacctcaagccagaatgc \\
RT-rrn-r & gctacaatggcgcatacaaa \\
RT-rrn-f & ttcatggagtcgagttgcag \\
\hline
\end{tabular}

Cloning 16S rRNA into pCIcheZ

Cloning 16S rRNA into pCIcheZ

RT qPCR primer for cheZ

RT qPCR primer for cheZ

RT qPCR primer for $c I$

RT qPCR primer for $c I$

RT qPCR primer for 16S rRNA (41)

RT qPCR primer for 16S rRNA (41)

Table S3 The parameters most used in the simulation

\begin{tabular}{lll}
\hline Parameters & Value & Comments \\
\hline$D_{\rho}$ & $450 \mu \mathrm{m}^{2} \mathrm{~s}^{-1}$ & The normal value $\left(200-1,000 \mu \mathrm{m}^{2} \mathrm{~s}^{-1}\right)(49-51)$ \\
$D_{\rho, 0}$ & $10 \mu \mathrm{m}^{2} \mathrm{~s}^{-1}$ & Fig. 1D shows it is almost zero \\
$D_{h}$ & $400 \mu \mathrm{m}^{2} \mathrm{~s}^{-1}$ & As small molecular diffusion $\left(100-1,000 \mu \mathrm{m}^{2} \mathrm{~s}^{-1}\right)(56,57)$ \\
$D_{n}$ & $800 \mu \mathrm{m}^{2} \mathrm{~s}^{-1}$ & As small molecular diffusion $\left(100-1,000 \mu \mathrm{m}^{2} \mathrm{~s}^{-1}\right)(56,57)$ \\
$\gamma$ & $0.7 \mathrm{~h}^{-1}$ & Measured shown in Fig. S16 \\
$\beta$ & $1.04 \mathrm{~h}^{-1}$ & AHL half-life ranging $10 \sim 1,000$ min $(39,59)$ \\
$m$ & 20 & Fig. 1D shows an abrupt fall of the cell motility \\
$n(\mathrm{t}=0)$ & $15 \times 10^{8} \mathrm{cells} \mathrm{ml}^{-1}$ & The saturated density cell as shown in Fig. S16 \\
$k_{n}$ & 1 & Rescaled with $n$ to the unit of $\rho$ \\
$K_{n}$ & $10^{9} \mathrm{cells} \mathrm{ml}^{-1}$ & Estimated from Fig. S16 \\
$K_{h}$ & $4 \times 10^{8} \mathrm{cells} \mathrm{ml}^{-1}$ & Estimated from Fig. 1D \\
\hline
\end{tabular}

